\title{
Platinum-Catalyzed Intramolecular Asymmetric Hydroarylation of Unactivated Alkenes with Indoles
}

Xiaoqing Han and Ross A. Widenhoefer

P. M. Gross Chemical Laboratory

Duke University

Durham, NC 27708-0346(USA)

e-mail: rwidenho@chem.duke.edu

\section{Supporting Information}

Experimental procedures, analytical and spectroscopic data, and copies of NMR spectra for products of catalytic hydroarylation (51 pages). 


\section{Experimental Section}

General Methods. Catalytic reactions were performed in a thick-walled pressure tube (Ace Glass) under an air atmosphere. NMR spectra were obtained on a Varian spectrometer operating at 400 $\mathrm{MHz}$ for ${ }^{1} \mathrm{H} \mathrm{NMR}$ and $100 \mathrm{MHz}$ for ${ }^{13} \mathrm{C} \mathrm{NMR}$ in $\mathrm{CDCl}_{3}$ unless otherwise noted. IR spectra were obtained on a Bomen MB-100 FT IR spectrometer. Gas chromatography was performed on a HewlettParkard 5890 gas chromatography equipped with a $25 \mathrm{~m}$ polydimethylsiloxane capillary column. Flash column chromatography was performed employing 200-400 mesh silica gel (EM). Thin layer chromatography (TLC) was performed on silica gel $60 \mathrm{~F}_{254}$ eluting with a 2:1 mixture of hexanes and ethyl acetate unless noted otherwise. Elemental analyses were performed by Complete Analysis Laboratories (Parsippany, NJ). 1,4-Dioxane (Acros, anhydrous), HCl solution (4.0 M, 1,4-dioxane, Aldrich), and anhydrous $\mathrm{MeOH}$ (Aldrich) were stored in an inert atmosphere glovebox. $\mathrm{PtCl}_{2}$, $\left[\mathrm{Pt}\left(\mathrm{CH}_{2}=\mathrm{CH}_{2}\right) \mathrm{Cl}_{2}\right]_{2},(S)$-Tol-BINAP, $(R)-3,5-X y l y l-B I N A P, \operatorname{Pt}(\mathrm{COD}) \mathrm{Cl}_{2}$ (Strem), ethyl 5-methoxy indole-2-carboxylate, 5-fluoro indole-2-carboxylate (Alfa Aesar), and ethyl indole-2-carboxylate (Aldrich) were used as received. (R)-3,5-i-Pr-MeOBIPHEP, and (S)-3,5-t-Bu-4-MeO-MeOBIPHEP [(S)-4] were provided by Dr. Rudolph Schmid (Hoffmann-LaRoche). Alkenyl indoles 3, 2-(1,1dimethyl-4-pentenyl)-1-methylindole, 2-(1,1-dimethyl-4-pentenyl)indole, and 2-(2-carbomethoxy-4pentenyl)-1-methylindole (7), ${ }^{\mathrm{S} 1} \quad\left[(S)-\mathrm{BINAP}_{\mathrm{PtCl}_{2}},{ }^{\mathrm{S} 2}\right.$ and ethyl 3,3-dimethyl-5-hexenate were synthesized employing literature procedures. ${ }^{\mathrm{S} 3}$

\section{Platinum Complexes}

[(S)-Tol-BINAP $] \mathbf{P t C l}_{2} \cdot[(S)$-Tol-BINAP $] \mathrm{PtCl}_{2}$ was isolated in $91 \%$ yield as a white solid from reaction of $(S)$-Tol-BINAP and $\mathrm{Pt}(\mathrm{COD}) \mathrm{Cl}_{2}$ employing a procedure similar to that reported for the synthesis of $[(S)-\mathrm{BINAP}] \mathrm{PtCl}_{2} .{ }^{\mathrm{S} 2}{ }^{1} \mathrm{H}$ NMR: $\oint 7.72-7.09(\mathrm{~m}, 22 \mathrm{H}), 6.74(\mathrm{~d}, J=8.8 \mathrm{~Hz}, 2 \mathrm{H}), 6.43$ (br s, 
$4 \mathrm{H}), 2.39$ (s, $6 \mathrm{H}), 1.98(\mathrm{~s}, 6 \mathrm{H}) .{ }^{31} \mathrm{P}\left\{{ }^{1} \mathrm{H}\right\} \mathrm{NMR}: \delta 9.6\left(\mathrm{~s},{ }^{1} J_{\mathrm{PPt}}=3664 \mathrm{~Hz}\right)$. Anal. Calcd. (found) for $\mathrm{C}_{48} \mathrm{H}_{40} \mathrm{Cl}_{2} \mathrm{P}_{2} \mathrm{Pt}: \mathrm{C}, 61.02$ (60.87); H, 4.27 (4.36).

$[(\boldsymbol{R})$-Xylyl-BINAP $] \mathbf{P t C l}_{2} \cdot[(R)$-Xylyl-BINAP $] \mathrm{PtCl}_{2}$ was isolated in $73 \%$ yield as a white solid employing a procedure similar to that reported for $[(S)$-BINAP $] \mathrm{PtCl}_{2}{ }^{\mathrm{S} 2}$ from reaction of $(R)$-XylylBINAP and $\mathrm{Pt}(\mathrm{COD}) \mathrm{Cl}_{2} .{ }^{1} \mathrm{H}$ NMR: $\oint 7.60-7.01(\mathrm{~m}, 20 \mathrm{H}), 6.50(\mathrm{~d}, J=8.4 \mathrm{~Hz}, 2 \mathrm{H}), 6.33(\mathrm{~s}, 2 \mathrm{H})$, 2.31 (s, $12 \mathrm{H}), 1.85$ (s, $12 \mathrm{H}) .{ }^{31} \mathrm{P}\left\{{ }^{1} \mathrm{H}\right\} \mathrm{NMR}: \delta 10.4\left(\mathrm{~s},{ }^{1} J_{\mathrm{PPt}}=3673 \mathrm{~Hz}\right.$ ). Anal. Calcd. (found) for $\mathrm{C}_{52} \mathrm{H}_{48} \mathrm{Cl}_{2} \mathrm{P}_{2} \mathrm{Pt}:$ C, 62.40 (62.21); H, 4.83 (5.02).

[(R)-3,5-i-Pr-MeOBIPHEP $]$ PtCl $_{2}$. [(R)-3,5-i-Pr-MeOBIPHEP $] \mathrm{PtCl}_{2}$ was isolated in $82 \%$ yield as a pale yellow solid employing a procedure similar to that reported for $\left[(S)-\mathrm{BINAP}_{\mathrm{PtCl}_{2}}{ }^{\mathrm{S} 2}\right.$ from reaction of $(R)-3,5-\mathrm{i}-\mathrm{Pr}-\mathrm{MeOBIPHEP}$ and $\mathrm{Pt}(\mathrm{COD}) \mathrm{Cl}_{2} .{ }^{1} \mathrm{H}$ NMR: $\oint 7.60-6.40(\mathrm{~m}, 18 \mathrm{H}), 3.50(\mathrm{~s}, 6 \mathrm{H})$, 2.90-2.78 (m, $8 \mathrm{H}),[1.24$ (d, $J=7.2 \mathrm{~Hz}), 1.21(\mathrm{~d}, J=6.8 \mathrm{~Hz}), 1.18(\mathrm{~d}, J=7.2 \mathrm{~Hz}), 1.17$ (d, $J=6.8 \mathrm{~Hz})$, $48 \mathrm{H}] .{ }^{31} \mathrm{P}\left\{{ }^{1} \mathrm{H}\right\} \mathrm{NMR}: \delta 11.5\left(\mathrm{~s},{ }^{1} \mathrm{JPP}_{\mathrm{Pt}}=3654 \mathrm{~Hz}\right.$ ). Anal. Calcd. (found) for $\mathrm{C}_{62} \mathrm{H}_{80} \mathrm{Cl}_{2} \mathrm{O}_{2} 2 \mathrm{P}_{2} \mathrm{Pt}: \mathrm{C}$, 62.83 (62.76); H, 6.80 (7.13).

$\left[(\boldsymbol{S})-3,5-t-\mathrm{Bu}^{4}-\mathrm{MeO}-\mathrm{MeOBIPHEP}\right] \mathbf{P t C l}_{2}\left\{[(\boldsymbol{S})-4] \mathrm{PtCl}_{2}\right\} \cdot \quad[(S)-4] \mathrm{PtCl}_{2}$ was isolated in $89 \%$ yield as a pale yellow solid employing a procedure similar to that reported for $\left[(R)-\mathrm{BINAP}_{\mathrm{PtCl}}{ }_{2}^{\mathrm{S} 2}\right.$ from reaction of (S)-4 and Pt(COD)Cl $2 .{ }^{1} \mathrm{H}$ NMR: $\oint 8.60-6.50(\mathrm{~m}, 14 \mathrm{H}), 3.68(\mathrm{~s}, 6 \mathrm{H}), 3.61(\mathrm{~s}, 12 \mathrm{H}), 1.37$ (s, $72 \mathrm{H}) .{ }^{31} \mathrm{P}\left\{{ }^{1} \mathrm{H}\right\}$ NMR: $\delta 11.4\left(\mathrm{~s},{ }^{1} J_{\mathrm{PPt}}=3664 \mathrm{~Hz}\right.$ ). Anal. Calcd. (found) for $\mathrm{C}_{74} \mathrm{H}_{104} \mathrm{Cl}_{2} \mathrm{O}_{6} \mathrm{P}_{2} \mathrm{Pt}: \mathrm{C}$, 62.70 (62.56); H, 7.39 (7.52).

\section{2-Alkenyl Indoles}

2-(2,2-dicarbomethoxy-4-pentenyl)-1-benzylindole (S1; Table 2, entry 1). Ethyl 1benzylindole-2-carboxylate $(\mathbf{S 2})^{\mathrm{S4}}$ was synthesized employing a literature procedure from reaction of ethyl indole-2-carboxylate and benzyl chloride. ${ }^{\mathrm{S} 4}$ A solution of $\mathbf{S 2}(1.40 \mathrm{~g}, 5.02 \mathrm{mmol})$ in THF (15 mL) 
was added slowly to a suspension of $\mathrm{LiAlH}_{4}(286 \mathrm{mg}, 7.53 \mathrm{mmol})$ in THF $(20 \mathrm{~mL})$ at $0{ }^{\circ} \mathrm{C}$. The reaction mixture was stirred at room temperature overnight, quenched with $1 \mathrm{~N} \mathrm{HCl}(30 \mathrm{~mL})$, and extracted with ether $(2 \times 50 \mathrm{~mL})$. The combined ether extracts were washed with water $(50 \mathrm{~mL})$, dried $\left(\mathrm{MgSO}_{4}\right)$, and concentrated under vacuum to give crude 2-hydroxymethyl-1-methylindole (S3), which was used for next step without further purification.

$n$-BuLi (2.5 M solution in hexanes, $1.96 \mathrm{~mL}, 4.89 \mathrm{mmol}$ ) was added to a solution of crude product of $\mathbf{S 3}$ in THF $(35 \mathrm{~mL})$ at $-78^{\circ} \mathrm{C}$. After stirring for $2 \mathrm{~min}$, methanesulfonyl chloride $(0.58 \mathrm{~g}, 5.1$ mmol) and $\operatorname{LiBr}(1.22 \mathrm{~g}, 14.0 \mathrm{mmol})$ were added sequentially. The resulting mixture was warmed to 0 ${ }^{\circ} \mathrm{C}$, stirred for $30 \mathrm{~min}$, and transferred via cannula to a solution of sodium dimethyl allylmalonate [generated from dimethyl allylmalonate $(0.88 \mathrm{~g}, 5.1 \mathrm{mmol})$ and $\mathrm{NaH}(0.123 \mathrm{~g}, 5.13 \mathrm{mmol})$ in THF (25 $\mathrm{mL})$ ]. The reaction mixture was stirred at room temperature for $4 \mathrm{~h}$, quenched with water, and extracted with ether $(2 \times 50 \mathrm{~mL})$. The combined organic extracts were washed (brine), dried $\left(\mathrm{MgSO}_{4}\right)$, and concentrated under vacuum. Column chromatography of the residue (hexanes-EtOAc $=5: 1$ ) gave S1 $(1.04 \mathrm{~g}, 53 \%$ from S2) as a white solid.

For S1: $\mathrm{mp} 116-118{ }^{\circ} \mathrm{C} . \mathrm{TLC}: R_{\mathrm{f}}=0.68 .{ }^{1} \mathrm{H}$ NMR: $\delta 7.55(\mathrm{~d}, J=8.0 \mathrm{~Hz}, 1 \mathrm{H}), 7.25-7.18(\mathrm{~m}$, $4 \mathrm{H}), 7.12-7.05(\mathrm{~m}, 2 \mathrm{H}), 6.89$ (d, J=8.0 Hz, $2 \mathrm{H}), 6.33(\mathrm{~s}, 1 \mathrm{H}), 5.61-5.50(\mathrm{~m}, 1 \mathrm{H}), 5.34(\mathrm{~s}, 2 \mathrm{H}), 5.02-$ $4.97(\mathrm{~m}, 2 \mathrm{H}), 3.69(\mathrm{~s}, 6 \mathrm{H}), 3.35(\mathrm{~s}, 2 \mathrm{H}), 2.82(\mathrm{~d}, J=7.2 \mathrm{~Hz}, 2 \mathrm{H}) .{ }^{13} \mathrm{C}\left\{{ }^{1} \mathrm{H}\right\} \mathrm{NMR}: \delta \quad 171.4,138.0$, $137.3,135.0,132.6,129.1,128.3,127.6,126.2,121.7,120.5,120.1,119.8,109.9,101.5,57.9,53.0$, 46.6, 37.3, 29.5. IR (neat, $\mathrm{cm}^{-1}$ ): 2954, 1735, 1461, 1224, 925. Anal. Calcd (found) for $\mathrm{C}_{24} \mathrm{H}_{25} \mathrm{NO}_{4}: \mathrm{C}_{\text {, }}$ 73.64 (73.50); H, 6.44 (6.69); N, 3.58 (3.63).

2-(2,2-dicarbomethoxy-4-pentenyl)-1-allylindole (S4; Table 2, entry 2): Ethyl 1-allylindole2-carboxylate $(\mathbf{S 5})^{\mathrm{S} 4}$ was synthesized employing a literature procedure from ethyl indole-2-carboxylate and allyl bromide. ${ }^{\mathrm{S} 4} \mathbf{S 4}$ was isolated in 27\% yield as a white solid from reaction of S5 and dimethyl 
allylmalonate employing a procedure similar to that used to synthesize $\mathbf{S 1} . \quad \mathrm{mp} 61-63{ }^{\circ} \mathrm{C}$. TLC: $R_{\mathrm{f}}=$ 0.63. ${ }^{1} \mathrm{H}$ NMR: $\delta 7.54(\mathrm{~d}, J=8.0 \mathrm{~Hz}, 1 \mathrm{H}), 7.24(\mathrm{~d}, J=8.0 \mathrm{~Hz}, 1 \mathrm{H}), 7.16(\mathrm{t}, J=7.6 \mathrm{~Hz}, 1 \mathrm{H}), 7.08(\mathrm{t}$, $J=7.2 \mathrm{~Hz}, 1 \mathrm{H}), 6.29(\mathrm{~s}, 1 \mathrm{H}), 5.95-5.86(\mathrm{~m}, 1 \mathrm{H}), 5.75-5.64(\mathrm{~m}, 1 \mathrm{H}), 5.12-5.05(\mathrm{~m}, 3 \mathrm{H}), 4.77-4.73(\mathrm{~m}$,

$3 \mathrm{H}), 3.74$ (s, $6 \mathrm{H}), 3.39$ (s, $2 \mathrm{H}), 2.88$ (d, $J=7.2 \mathrm{~Hz}, 2 \mathrm{H}) .{ }^{13} \mathrm{C}\left\{{ }^{1} \mathrm{H}\right\}$ NMR: $\delta \quad 171.5,136.9,134.8$, 133.5, 132.6, 128.2, 121.5, 120.5, 11993, 119.88, 116.6, 109.7, 101.2, 57.9, 53.0, 45.4, 37.3, 29.3. IR (neat, $\mathrm{cm}^{-1}$ ): 2951, 1736, 1462, 1435, 1290, 924. Anal. Calcd (found) for $\mathrm{C}_{20} \mathrm{H}_{23} \mathrm{NO}_{4}: \mathrm{C}, 70.36$ (70.51); H, 6.79 (6.97); N, 4.10 (4.23).

2-(2,2-dicarbomethoxy-4-pentenyl)-1-methyl-5-methoxyindole (S6; Table 2, entry 3). Ethyl 5-methoxy-1-methylindole-2-carboxylate ${ }^{\mathrm{S} 5}$ was synthesized employing a literature procedure from ethyl 5-methoxy indole-2-carboxylate and MeI. ${ }^{\mathrm{S} 4}$ S6 was isolated in 26\% yield as a yellow solid from reaction of ethyl 5-methoxy-1-methyl-indole-2-carboxylate and dimethyl allylmalonate employing a procedure similar to that used to synthesize S1. Yellow solid. mp 89-91 ${ }^{\circ} \mathrm{C}$. TLC: $R_{\mathrm{f}}=0.56$. ${ }^{1} \mathrm{H}$ NMR: $\delta 7.15(\mathrm{~d}, J=8.4 \mathrm{~Hz}, 1 \mathrm{H}), 7.00(\mathrm{~s}, 1 \mathrm{H}), 6.85-6.82(\mathrm{~m}, 1 \mathrm{H}), 6.18(\mathrm{br} \mathrm{s}, 1 \mathrm{H}), 5.78-5.67$ (m, 1 H), 5.14-5.07 (m, $2 \mathrm{H}), 3.84(\mathrm{~s}, 3 \mathrm{H}), 3.73(\mathrm{~s}, 6 \mathrm{H}), 3.63(\mathrm{~s}, 3 \mathrm{H}), 3.41(\mathrm{~s}, 2 \mathrm{H}), 2.79(\mathrm{~d}, J=7.2 \mathrm{~Hz}, 2 \mathrm{H})$. ${ }^{13} \mathrm{C}\left\{{ }^{1} \mathrm{H}\right\} \mathrm{NMR}: \quad \delta \quad 171.5,154.4,135.5,132.9,132.7,128.3,119.8,111.6,110.1,102.3,101.0,58.3$, 56.2, 53.0, 37.3, 30.1, 29.6. IR (neat, $\mathrm{cm}^{-1}$ ): 2949, 1734, 1483, 1433, 1213. Anal. Calcd (found) for $\mathrm{C}_{19} \mathrm{H}_{23} \mathrm{NO}_{5}: \mathrm{C}, 66.07$ (65.86); H, 6.71 (6.85); N, 4.06 (3.88).

2-(2,2-dicarbomethoxy-4-pentenyl)-1-methyl-5-fluoroindole (S7; Table 2, entry 4). Ethyl 5fluoro-1-methyl- $1 H$-indole-2-carboxylate ${ }^{\mathrm{S} 4}$ was synthesized employing a literature procedure from ethyl 5-fluoro indole-2-carboxylate and MeI. ${ }^{\mathrm{S}}$ S7 was isolated in $62 \%$ yield as a pale yellow solid from reaction of ethyl 5-fluoro-1-methylindole-2-carboxylate and dimethyl allylmalonate employing a procedure similar to that used to synthesize S1. TLC: $R_{\mathrm{f}}=0.64 .{ }^{1} \mathrm{H}$ NMR: $\delta 7.18-7.14(\mathrm{~m}, 2 \mathrm{H}), 6.94-$ $6.88(\mathrm{~m}, 1 \mathrm{H}), 6.23(\mathrm{~s}, 1 \mathrm{H}), 5.78-5.68(\mathrm{~m}, 1 \mathrm{H}), 5.16-5.08(\mathrm{~m}, 2 \mathrm{H}), 3.74(\mathrm{~s}, 6 \mathrm{H}), 3.65(\mathrm{~s}, 3 \mathrm{H}), 3.42(\mathrm{~s}, 2$ 
H), $2.79(\mathrm{~d}, J=7.6 \mathrm{~Hz}, 2 \mathrm{H}) .{ }^{13} \mathrm{C}\left\{{ }^{1} \mathrm{H}\right\}$ NMR: $\delta \quad 171.3,158.2(\mathrm{~d}, J=232.5 \mathrm{~Hz}), 136.7,134.2,132.5$, $128.2(\mathrm{~d}, J=10.2 \mathrm{~Hz}), 119.9,109.9(\mathrm{~d}, J=9.8 \mathrm{~Hz}), 109.6(\mathrm{~d}, J=26.1 \mathrm{~Hz}), 105.1(\mathrm{~d}, J=23.1 \mathrm{~Hz}), 101.3$ (d, $J=4.6 \mathrm{~Hz}$ ), 58.2, 53.0, 37.3, 30.2, 29.6. IR (neat, $\mathrm{cm}^{-1}$ ): 2952, 1735, 1483, 1435, 1214, 1136. Anal. Calcd (found) for $\mathrm{C}_{18} \mathrm{H}_{20} \mathrm{FNO}_{4}$ : C, 64.85 (64.85); H, 6.05 (6.29); N, 4.20 (4.19).

2-(2,2-dihydroxymethyl-4-pentenyl)-1-methylindole (S8; Table 2, entry 5). A solution of $3^{\mathrm{S} 1}$ $(1.30 \mathrm{~g}, 4.13 \mathrm{mmol})$ in THF $(15 \mathrm{~mL})$ was added slowly to a suspension of $\mathrm{LiAlH}_{4}(471 \mathrm{mg}, 12.4 \mathrm{mmol})$ in $\operatorname{THF}(20 \mathrm{~mL})$ at $0{ }^{\circ} \mathrm{C}$. The reaction mixture was stirred at room temperature overnight, quenched with $1 \mathrm{~N} \mathrm{HCl}(50 \mathrm{~mL})$, and extracted with ether $(2 \times 50 \mathrm{~mL})$. The combined ether extracts were washed with water $(50 \mathrm{~mL})$, dried $\left(\mathrm{MgSO}_{4}\right)$, and concentrated under vacuum to give an oil that solidified upon addition of hexanes/EtOAc (3:1, $3 \mathrm{~mL})$. The solid was washed with hexanes $(3 \mathrm{~mL})$ to give $\mathbf{S 8}(0.54 \mathrm{~g}$, $50 \%$ yield) as a white solid. $\mathrm{mp} 96-97.5{ }^{\circ} \mathrm{C} . \quad \mathrm{TLC}: R_{\mathrm{f}}=0.14 .{ }^{1} \mathrm{H}$ NMR: $\delta 7.55(\mathrm{~d}, J=7.6 \mathrm{~Hz}, 1 \mathrm{H})$, $7.30(\mathrm{~d}, J=8.0 \mathrm{~Hz}, 1 \mathrm{H}), 7.19(\mathrm{t}, J=7.2 \mathrm{~Hz}, 1 \mathrm{H}), 7.11(\mathrm{t}, J=6.8 \mathrm{~Hz}, 1 \mathrm{H}), 6.37$ (br s, $1 \mathrm{H}), 5.96-5.85$ (m, 1 H), 5.18-5.14 (m, 2 H), 3.74 (s, 3 H), 3.63 (ABq, J=10.8 Hz, 4 H), 2.88 (s, 2 H), 2.27 (br s, 2 H), $2.18(\mathrm{~d}, J=7.6 \mathrm{~Hz}, 2 \mathrm{H}) .{ }^{13} \mathrm{C}\left\{{ }^{1} \mathrm{H}\right\}$ NMR: $\delta \quad 137.7,137.3,134.2,128.2,121.2,120.1,119.9,118.9$, 109.6, 102.0, 67.8, 43.9, 37.5, 30.4, 28.8. IR (neat, $\mathrm{cm}^{-1}$ ): 3384, 2929, 1466, 1317, 1024, 918. Anal. Calcd (found) for $\mathrm{C}_{16} \mathrm{H}_{21} \mathrm{NO}_{2}: \mathrm{C}, 74.10$ (74.24); $\mathrm{H}, 8.16$ (8.09); N, 5.40 (5.65).

2-[2,2-di-(2,2-Dimethyl-propionyloxymethyl)-4-pentenyl]-1-methylindole (S9; Table 2, entry 6). Pivaloyl chloride $(0.37 \mathrm{~mL}, 3.00 \mathrm{mmol})$ was added slowly to a solution of $\mathbf{S 8}$ (312 $\mathrm{mg}, 1.20$ $\mathrm{mmol}), \mathrm{Et}_{3} \mathrm{~N}(0.50 \mathrm{~mL}, 3.6 \mathrm{mmol})$, and DMAP $(73 \mathrm{mg}, 0.6 \mathrm{mmol})$ in $\mathrm{CH}_{2} \mathrm{Cl}_{2}(20 \mathrm{~mL})$ at $0{ }^{\circ} \mathrm{C}$. The reaction mixture was stirred at room temperature overnight, quenched with brine $(20 \mathrm{~mL})$, extracted with $\mathrm{CH}_{2} \mathrm{Cl}_{2}(30 \mathrm{~mL})$. The organic layer was dried $\left(\mathrm{MgSO}_{4}\right)$ and concentrated under vacuum. Column chromatography of the residue (hexanes-EtOAc $=5: 1)$ gave $\mathbf{S 9}(0.47 \mathrm{~g}, 90 \%$ yield) as a viscous colorless oil. TLC: $R_{\mathrm{f}}=0.85 .{ }^{1} \mathrm{H}$ NMR: $\delta 7.56(\mathrm{~d}, J=7.6 \mathrm{~Hz}, 1 \mathrm{H}), 7.28(\mathrm{~d}, J=8.0 \mathrm{~Hz}, 1 \mathrm{H}), 7.20(\mathrm{t}, J$ 
$=7.2 \mathrm{~Hz}, 1 \mathrm{H}), 7.11(\mathrm{t}, J=7.6 \mathrm{~Hz}, 1 \mathrm{H}), 6.32(\mathrm{~s}, 2 \mathrm{H}), 5.87-5.77(\mathrm{~m}, 1 \mathrm{H}), 5.18-5.10(\mathrm{~m}, 2 \mathrm{H}), 4.03(\mathrm{~s}, 4$ H), 3.70 (s, $3 \mathrm{H}), 2.94$ (s, $2 \mathrm{H}), 2.26$ (d, $J=7.2 \mathrm{~Hz}, 2 \mathrm{H}), 1.24(\mathrm{~s}, 9 \mathrm{H}) .{ }^{13} \mathrm{C}\left\{{ }^{1} \mathrm{H}\right\}$ NMR: $\delta 178.3,137.7$, $135.4,132.7,128.1,121.4,120.2,120.0,119.8,109.6,102.8,65.9,42.2,39.3,37.4,30.2,29.2,27.5$. IR (neat, $\mathrm{cm}^{-1}$ ): 2972, 1730, 1479, 1282, 1150. Anal. Calcd (found) for $\mathrm{C}_{16} \mathrm{H}_{21} \mathrm{NO}_{2}: \mathrm{C}, 73.03$ (72.97); $\mathrm{H}$, $8.72(8.78) ; \mathrm{N}, 3.28(3.52)$.

2-(2,2-dicarbo-t-butoxy-4-pentenyl)-1-methylindole (Table 2, entry 7). 2-(2,2-Dicarbo-tbutoxy-4-pentenyl)-1-methylindole was isolated in 50\% yield as a white solid from reaction of 2hydroxymethyl-1-methylindol ${ }^{\mathrm{S} 1}$ and di-t-butyl allylmalonate employing a procedure similar to that used to synthesize S1. $\operatorname{mp} 45-47^{\circ} \mathrm{C}$. TLC: $R_{\mathrm{f}}=0.63 .{ }^{1} \mathrm{H}$ NMR: $\delta 7.51(\mathrm{~d}, J=8.0 \mathrm{~Hz}, 1 \mathrm{H}), 7.24(\mathrm{~d}, J=8.4$ Hz, $1 \mathrm{H}), 7.14$ (t, $J=7.2 \mathrm{~Hz}, 1 \mathrm{H}), 7.04$ (t, $J=7.2 \mathrm{~Hz}, 1 \mathrm{H}), 6.32$ (br s, $1 \mathrm{H}), 5.74-5.63$ (m, $1 \mathrm{H}), 5.09$ 5.00 (m, $2 \mathrm{H}), 3.66(\mathrm{~s}, 3 \mathrm{H}), 3.31$ (s, $2 \mathrm{H}), 2.75$ (d, $J=7.2 \mathrm{~Hz}, 2 \mathrm{H}), 1.42(\mathrm{~s}, 18 \mathrm{H}) .{ }^{13} \mathrm{C}\left\{{ }^{1} \mathrm{H}\right\}$ NMR: $\delta$ 170.3, 137.3, 135.6, 133.0, 128.2, 121.1, 120.3, 119.6, 119.4, 109.2, 101.0, 82.1, 58.4, 37.1, 30.1, 28.9, 28.2. IR (neat, $\mathrm{cm}^{-1}$ ): 2977, 2939, 1727, 1468, 1368, 1224, 1149, 920. Anal. Calcd (found) for $\mathrm{C}_{23} \mathrm{H}_{33} \mathrm{NO}_{4}$ : C, 72.15 (72.25); H, 8.33 (8.41); N, 3.51 (3.67).

2-(2,2-dimethyl-4-pentenyl)-1-methylindole (S10; Table 2, entry 8). $n$-BuLi (2.5 M solution in hexanes, $8.92 \mathrm{~mL}, 22.3 \mathrm{mmol}$ ) was added dropwise to a solution of $N$-trimethylsilyl-o-toluidine ${ }^{\mathrm{S} 6}$ $(1.81 \mathrm{~g}, 10.13 \mathrm{mmol})$ in hexanes $(50 \mathrm{~mL})$ at $0{ }^{\circ} \mathrm{C}$. The resulting orange solution was refluxed for $5 \mathrm{~h}$, cooled to $-78^{\circ} \mathrm{C}$, and treated with a solution of ethyl 3,3-dimethyl-5-hexenate ${ }^{\mathrm{S} 3}(2.07 \mathrm{~g}, 12.16 \mathrm{mmol})$ in THF $(10 \mathrm{~mL})$. The resulting mixture was warmed to room temperature, stirred for $0.5 \mathrm{~h}$, and quenched with brine. The aqueous layer was extracted with ether $(2 \times 50 \mathrm{~mL})$. The combined organic extracts were dried $\left(\mathrm{MgSO}_{4}\right)$ and concentrated under vacuum. Column chromatography of the residue (hexanes$\mathrm{CH}_{2} \mathrm{Cl}_{2}=10: 1$ ) gave an oil that was chromatographed a second time (hexanes-EtOAc $=20: 1$ ) to give 2(2,2-dimethyl-4-pentenyl)indole (S11, $0.79 \mathrm{~g}, 37 \%)$ as a pale yellow oil. 
A solution of $\mathbf{S 1 1}(0.74 \mathrm{~g}, 3.5 \mathrm{mmol})$ in DMF $(9 \mathrm{~mL})$ was added slowly to a suspension of $\mathrm{NaH}$ ( $88 \mathrm{mg}, 3.65 \mathrm{mmol})$ in DMF $(5 \mathrm{~mL})$ at $0{ }^{\circ} \mathrm{C}$. The mixture was stirred at $0{ }^{\circ} \mathrm{C}$ for $20 \mathrm{~min}$ and MeI $(0.518$ $\mathrm{g}, 3.65 \mathrm{mmol}$ ) was added. The reaction mixture was stirred at room temperature for 1 hour, quenched with brine $(50 \mathrm{~mL})$, extracted with ether $(2 \times 40 \mathrm{~mL})$. The combined ether extracts were washed with water $(50 \mathrm{~mL})$, dried $\left(\mathrm{MgSO}_{4}\right)$, and concentrated under vacuum. Column chromatography of the residue (hexanes-EtOAc $=25: 1)$ gave $\mathbf{S 1 0}(0.74 \mathrm{~g}, 94 \%)$ as a pale yellow oil.

For S11: TLC (hexanes-EtOAc $=5: 1): R_{\mathrm{f}}=0.50 .{ }^{1} \mathrm{H}$ NMR: $\delta 7.85(\mathrm{br} \mathrm{s}, 1 \mathrm{H}), 7.59(\mathrm{~d}, J=7.6$ $\mathrm{Hz}, 1 \mathrm{H}), 7.33$ (d, $J=7.6 \mathrm{~Hz}, 1 \mathrm{H}), 7.17$ (t, $J=7.2 \mathrm{~Hz}, 1 \mathrm{H}), 7.12$ (t, $J=7.2 \mathrm{~Hz}, 1 \mathrm{H}), 6.28(\mathrm{~s}, 1 \mathrm{H})$, 6.00-5.89 (m, $1 \mathrm{H}), 5.18-5.10(\mathrm{~m}, 2 \mathrm{H}), 2.64(\mathrm{~s}, 2 \mathrm{H}), 2.08(\mathrm{~d}, J=7.2 \mathrm{~Hz}, 2 \mathrm{H}), 1.00(\mathrm{~s}, 6 \mathrm{H}) .{ }^{13} \mathrm{C}\left\{{ }^{1} \mathrm{H}\right\}$ NMR: $\delta 137.2,136.0,135.9,129.1,121.3,120.1,119.9,117.8,110.6,102.5,46.7,41.2,34.9,27.4$. IR (neat, $\mathrm{cm}^{-1}$ ): $3409,2958,2926,1455,1287,913$. HRMS Calcd (found) for $\mathrm{C}_{15} \mathrm{H}_{19} \mathrm{~N}\left(\mathrm{M}^{+}\right): 213.1517$ (213.1519).

For S10: TLC (hexanes-EtOAc $=5: 1): R_{\mathrm{f}}=0.67 .{ }^{1} \mathrm{H}$ NMR: $\delta 7.55(\mathrm{~d}, J=7.6 \mathrm{~Hz}, 1 \mathrm{H}), 7.27$ $(\mathrm{d}, J=8.0 \mathrm{~Hz}, 1 \mathrm{H}), 7.16(\mathrm{t}, J=7.2 \mathrm{~Hz}, 1 \mathrm{H}), 7.08(\mathrm{t}, J=7.6 \mathrm{~Hz}, 1 \mathrm{H}), 6.29(\mathrm{~s}, 1 \mathrm{H}), 5.95-5.84(\mathrm{~m}, 1 \mathrm{H})$, 5.11-5.04 (m, $2 \mathrm{H}), 3.67$ (s, $3 \mathrm{H}), 2.68(\mathrm{~s}, 2 \mathrm{H}), 2.10(\mathrm{~d}, J=7.6 \mathrm{~Hz}, 2 \mathrm{H}), 0.94(\mathrm{~s}, 6 \mathrm{H}) .{ }^{13} \mathrm{C}\left\{{ }^{1} \mathrm{H}\right\} \mathrm{NMR}$ : $\delta 138.7,137.6,135.6,128.2,120.8,120.0,119.7,117.9,109.4,102.1,47.2,38.4,35.6,30.5,27.0 . \quad$ IR (neat, $\mathrm{cm}^{-1}$ ): 3056, 2957, 1467, 1340, 1317, 913. Anal. Calcd (found) for $\mathrm{C}_{16} \mathrm{H}_{21} \mathrm{~N}$ : C, 84.53 (84.63); H, 9.31 (9.35); N, $6.16(6.34)$.

2-(2,2-dicarbomethoxy-5-hexenyl)-1-methylindole $\quad$ (Table $\quad 2, \quad$ entry $\quad 11) . \quad 2-(2,2-$ Dicarbomethoxy-5-hexenyl)-1-methylindole was isolated in 35\% yield as a viscous pale yellow oil from reaction of 2-hydroxymethyl-1-methylindole ${ }^{\mathrm{S} 1}$ and dimethyl 3-butenylmalonate employing a procedure similar to that used to synthesize S1. TLC: $R_{\mathrm{f}}=0.47 .{ }^{1} \mathrm{H}$ NMR: $\delta 7.52(\mathrm{~d}, J=8.0 \mathrm{~Hz}, 1 \mathrm{H}), 7.27(\mathrm{~d}, J$ $=8.0 \mathrm{~Hz}, 1 \mathrm{H}), 7.18(\mathrm{dt}, J=1.2,7.6 \mathrm{~Hz}, 1 \mathrm{H}), 7.07(\mathrm{dt}, J=1.2,7.6 \mathrm{~Hz}, 1 \mathrm{H}), 6.24(\mathrm{~s}, 1 \mathrm{H}), 5.81-5.71(\mathrm{~m}$, 
$1 \mathrm{H})$, 5.06-4.96 (m, $2 \mathrm{H}), 3.74(\mathrm{~s}, 6 \mathrm{H}), 3.67(\mathrm{~s}, 3 \mathrm{H}), 3.46(\mathrm{~s}, 2 \mathrm{H}), 2.15-2.10(\mathrm{~m}, 2 \mathrm{H}), 2.07-2.01(\mathrm{~m}, 2$ H). ${ }^{13} \mathrm{C}\left\{{ }^{1} \mathrm{H}\right\}$ NMR: $\delta 171.7,137.5,137.4,134.9,128.0,121.4,120.3,119.8,115.6,109.4,101.4,57.9$, 52.9, 31.8, 29.9, 29.7, 29.0. IR (neat, $\mathrm{cm}^{-1}$ ): 2952, 1732, 1464, 1437, 1204, 917. HRMS Calcd (found) for $\mathrm{C}_{19} \mathrm{H}_{23} \mathrm{NO}_{4}\left(\mathrm{M}^{+}\right): 329.1627$ (329.1624).

\section{Polycyclic Indoles}

Complete optimization for the conversion of $\mathbf{3}$ to $\mathbf{5}$ is depicted in Tables S1 and S2.

2,2-Dicarbomethoxy-4,9-dimethyl-1,3,4,9-tetrahydrocarbazole (5). ${ }^{\text {S1 }}$ A suspension of 3 (67 $\mathrm{mg}, 0.21 \mathrm{mmol}),[(S)-4] \mathrm{PtCl}_{2}\left(30 \mathrm{mg}, 2.1 \times 10^{-2} \mathrm{mmol}\right)$, and AgOTf $\left(5 \mathrm{mg}, 2.1 \times 10^{-2} \mathrm{mmol}\right)$ in $\mathrm{MeOH}$ $(0.82 \mathrm{~mL})$ was stirred at $60{ }^{\circ} \mathrm{C}$ for 20 hours, cooled to room temperature, and filtered through a plug of silica gel. The silica gel was eluted with ether, the filtrate and eluant were combined, and concentrated under vacuum. Chromatography of the residue (hexanes-EtOAc $=10: 1)$ gave $5(62 \mathrm{mg}, 93 \%, 90 \%$ ee) as a white solid. The enantiomeric excess of $\mathbf{5}$ was determined by HPLC analysis using a Chiralpak AD-H column (Figure S1).

All remaining polycyclic indole derivatives were isolated from the corresponding 2alkenylindoles employing a similar procedure to that for the synthesis of $\mathbf{5}$ unless noted otherwise. 1,1,4,9-Tetramethyl-2,3,4,9-tetrahydrocarbazole (Table 2, entry 9), 1,1,4-trimethyl-2,3,4,9tetrahydrocarbazole (Table 2, entry 10), and $\mathbf{8}$ have been previously characterized. ${ }^{\mathrm{S} 1}$ The enantiomeric excess of polycyclic indole derivatives were determined by HPLC analysis using a Chiralpak AD-H column (250 mm) (See Figures S2-S13).

2,2-Dicarbomethoxy-4-methyl-9-benzyl-1,3,4,9-tetrahydrocarbazole (S12; Table 2, entry 1). White solid. $\mathrm{mp} 44-48{ }^{\circ} \mathrm{C}$. TLC: $R_{\mathrm{f}}=0.62 .{ }^{1} \mathrm{H}$ NMR (Figure S14): $\delta 7.63(\mathrm{~d}, J=8.0 \mathrm{~Hz}, 1 \mathrm{H}$ ), 7.29-7.18 (m, $4 \mathrm{H}), 7.12-7.02(\mathrm{~m}, 4 \mathrm{H}), 5.28$ (s, $2 \mathrm{H}), 3.76$ (s, $3 \mathrm{H}), 3.60(\mathrm{~s}, 3 \mathrm{H}), 3.45(\mathrm{~d}, J=16.4 \mathrm{~Hz}, 1$ 
H), 3.27-3.21 (m, $1 \mathrm{H}), 3.04(\mathrm{~d}, J=16.0 \mathrm{~Hz}, 1 \mathrm{H}), 2.73(\mathrm{dd}, J=6.0,13.6 \mathrm{~Hz}, 1 \mathrm{H}), 1.88(\mathrm{dd}, J=10.4$, $13.2 \mathrm{~Hz}, 1 \mathrm{H}), 1.49(\mathrm{~d}, J=6.8,3 \mathrm{H}) .{ }^{13} \mathrm{C}\left\{{ }^{1} \mathrm{H}\right\}$ NMR (Figure S15): $\delta \quad 172.5,171.0,138.0,137.5,132.3$, $129.0,127.6,126.8,126.4,121.3,119.8,119.3,113.5,109.7,54.8,53.2,53.0,46.7,38.7,28.5,26.4$, 21.6. IR (neat, $\mathrm{cm}^{-1}$ ): 2952, 1735, 1465, 1250, 1028. Anal. Calcd (found) for $\mathrm{C}_{24} \mathrm{H}_{25} \mathrm{NO}_{4}: \mathrm{C}, 73.64$ (73.76); H, 6.44 (6.83); N, $3.58(3.66)$.

\section{2,2-Dicarbomethoxy-4-methyl-9-allyl-1,3,4,9-tetrahydrocarbazole (S13; Table 2, entry 2).}

Colorless oil. TLC: $R_{\mathrm{f}}=0.52 .{ }^{1} \mathrm{H}$ NMR (Figure S16): $\delta 7.60(\mathrm{~d}, J=8.0 \mathrm{~Hz}, 1 \mathrm{H}), 7.23(\mathrm{~d}, J=8.0 \mathrm{~Hz}$, $1 \mathrm{H}), 7.14(\mathrm{t}, J=8.4 \mathrm{~Hz}, 1 \mathrm{H}), 7.06(\mathrm{t}, J=8.0 \mathrm{~Hz}, 1 \mathrm{H}), 5.97-5.88(\mathrm{~m}, 1 \mathrm{H}), 5.13(\mathrm{dd}, J=1.2,10.8 \mathrm{~Hz}, 1$ H), $4.86(\mathrm{dd}, J=1.2,16.8 \mathrm{~Hz}, 1 \mathrm{H}), 4.66(\mathrm{t}, J=2.4 \mathrm{~Hz}, 2 \mathrm{H}), 3.78(\mathrm{~s}, 3 \mathrm{H}), 3.63(\mathrm{~s}, 3 \mathrm{H}), 3.45$ (d, $J=$ $16.0 \mathrm{~Hz}, 1 \mathrm{H}), 3.22-3.12(\mathrm{~m}, 1 \mathrm{H}), 3.06(\mathrm{dd}, J=2.0,16.0 \mathrm{~Hz}, 1 \mathrm{H}), 2.71(\mathrm{ddd}, J=2.0,5.6,13.4 \mathrm{~Hz}, 1$ $\mathrm{H}), 1.87(\mathrm{dd}, J=10.0,13.4 \mathrm{~Hz}, 1 \mathrm{H}), 1.45(\mathrm{~d}, J=6.8 \mathrm{~Hz}, 3 \mathrm{H}) .{ }^{13} \mathrm{C}\left\{{ }^{1} \mathrm{H}\right\}$ NMR (Figure S17): $\delta 172.5$, 171.0, 137.2, 133.5, 132.2, 126.7, 121.1, 119.8, 119.2, 116.6, 113.2, 109.4, 54.8, 53.2, 53.0, 45.4, 38.7, 28.4, 26.3, 21.6. IR (neat, $\mathrm{cm}^{-1}$ ): 2953, 1735, 1464, 1250, 1199, 922. Anal. Calcd (found) for $\mathrm{C}_{20} \mathrm{H}_{23} \mathrm{NO}_{4}$ : C, 70.36 (70.30); H, 6.79 (6.57); N, 4.10 (4.05).

2,2-Dicarbomethoxy-4,9-dimethyl-6-methoxy-1,3,4,9-tetrahydrocarbazole (S14; Table 2, entry 3). White solid. mp 108-109 ${ }^{\circ} \mathrm{C}$. TLC: $R_{\mathrm{f}}=0.45 .{ }^{1} \mathrm{H}$ NMR (Figure S18): $\delta 7.16(\mathrm{~d}, J=8.8 \mathrm{~Hz}$, $1 \mathrm{H}), 7.07$ (d, $J=2.4 \mathrm{~Hz}, 1 \mathrm{H}), 6.84(\mathrm{dd}, J=8.8 \mathrm{~Hz}, 1 \mathrm{H}), 3.86(\mathrm{~s}, 3 \mathrm{H}), 3.81(\mathrm{~s}, 3 \mathrm{H}), 3.68(\mathrm{~s}, 3 \mathrm{H})$, $3.63(\mathrm{~s}, 3 \mathrm{H}), 3.49$ (d, $J=16.0 \mathrm{~Hz}, 1 \mathrm{H}),[3.15-3.07$ (m), 3.09 (dd, $J=2.0,16.0 \mathrm{~Hz}), 2 \mathrm{H}], 2.71(\mathrm{dd}, J=$ 4.4, $13.4 \mathrm{~Hz}, 1 \mathrm{H}), 1.87(\mathrm{dd}, J=10.4,13.2 \mathrm{~Hz}, 1 \mathrm{H}), 1.45(\mathrm{~d}, J=6.8 \mathrm{~Hz}, 3 \mathrm{H}) .{ }^{13} \mathrm{C}\left\{{ }^{1} \mathrm{H}\right\}$ NMR (Figure S19): $\delta 172.5,171.1,153.8,133.3,126.7,112.4,110.4,109.6,102.7,56.4,54.7,53.2,53.1,38.7,29.6$, 28.6, 26.3, 21.4. IR (neat, $\mathrm{cm}^{-1}$ ): 2954, 1737, 1485, 1248, 1222, 1039. Anal. Calcd (found) for $\mathrm{C}_{19} \mathrm{H}_{23} \mathrm{NO}_{5}: \mathrm{C}, 66.07$ (66.21); H, 6.71 (6.80); N, 4.06 (4.14). 


\section{2,2-Dicarbomethoxy-4,9-dimethyl-6-flouro-1,3,4,9-tetrahydrocarbazole (S15; Table 2,}

entry 4). White solid. mp $137-138{ }^{\circ} \mathrm{C} . \mathrm{TLC}: R_{\mathrm{f}}=0.55 .{ }^{1} \mathrm{H}$ NMR (Figure S20): $\delta$ 7.24-7.21 (m, $1 \mathrm{H}$ ), 7.17-7.14 (m, $1 \mathrm{H}), 6.92-6.86(\mathrm{~m}, 1 \mathrm{H}), 3.81(\mathrm{~s}, 3 \mathrm{H}), 3.68(\mathrm{~s}, 3 \mathrm{H}), 3.64(\mathrm{~s}, 3 \mathrm{H}), 3.50(\mathrm{~d}, J=16.0 \mathrm{~Hz}, 1$ H), [3.19-3.05 (m), $3.08(\mathrm{~d}, J=8.0 \mathrm{~Hz}), 2 \mathrm{H}], 2.70(\mathrm{dd}, J=5.2,13.4 \mathrm{~Hz}, 1 \mathrm{H}), 1.86(\mathrm{dd}, J=10.4,13.4$ $\mathrm{Hz}, 1 \mathrm{H}), 1.42(\mathrm{~d}, J=6.8 \mathrm{~Hz}, 3 \mathrm{H}) .{ }^{13} \mathrm{C}\left\{{ }^{1} \mathrm{H}\right\}$ NMR (Figure S21): $\delta 172.4,171.1,157.7(\mathrm{~d}, J=231.7$ Hz), 134.5, 134.4, 126.6 (d, $J=9.5 \mathrm{~Hz}), 112.9$ (d, $J=4.5 \mathrm{~Hz}), 109.5$ (d, $J=9.9 \mathrm{~Hz}), 108.9$ (d, $J=26.2$ $\mathrm{Hz}), 104.8(\mathrm{~d}, \mathrm{~d}, J=23.5 \mathrm{~Hz}), 54.7,53.3,53.2,38.6,29.7,28.6,26.2,21.3$. IR (neat, $\mathrm{cm}^{-1}$ ): 2954, 1736, 1484, 1246, 1199. Anal. Calcd (found) for $\mathrm{C}_{18} \mathrm{H}_{20} \mathrm{FNO}_{4}$ : C, 64.85 (64.95); H, 6.05 (6.16); N, 4.20 (4.27).

\section{2,2-Dihydroxymethyl-4,9-dimethyl-1,3,4,9-tetrahydrocarbazole (S16; Table 2, entry 5).}

Pale yellow solid. mp $145-147{ }^{\circ} \mathrm{C}$. TLC: $R_{\mathrm{f}}=0.07 .{ }^{1} \mathrm{H}$ NMR (Figure S22): $\delta 7.60(\mathrm{~d}, J=7.6 \mathrm{~Hz}, 1$ H), $7.24(\mathrm{~d}, J=8.4 \mathrm{~Hz}, 1 \mathrm{H}), 7.13(\mathrm{t}, J=7.2 \mathrm{~Hz}, 1 \mathrm{H}), 7.04(\mathrm{t}, J=7.6 \mathrm{~Hz}, 1 \mathrm{H}),[3.70-3.59(\mathrm{~m}), 3.59(\mathrm{~s})$, $7 \mathrm{H}], 3.01-2.77$ (m, $1 \mathrm{H}), 2.77$ (d, $J=16.8 \mathrm{~Hz}, 1 \mathrm{H}), 2.44$ (dd, $J=2.0,16.4 \mathrm{~Hz}, 1 \mathrm{H}), 2.26($ br s, $2 \mathrm{H})$, $1.94(\mathrm{dd}, J=5.2,13.6 \mathrm{~Hz}, 1 \mathrm{H}), 1.42(\mathrm{~d}, J=6.8 \mathrm{~Hz}, 3 \mathrm{H}), 1.22(\mathrm{dd}, J=10.8,13.0 \mathrm{~Hz}, 1 \mathrm{H}) .{ }^{13} \mathrm{C}\left\{{ }^{1} \mathrm{H}\right\}$ NMR (Figure S23): $\delta \quad 137.8,134.0,126.7,120.8,119.8,119.0,113.0,109.1,72.6,67.1,40.3,38.4$, 29.4, 26.8, 25.7, 21.9. IR (neat, $\mathrm{cm}^{-1}$ ): 3294, 2929, 1467, 1420, 1322, 1026. Anal. Calcd (found) for $\mathrm{C}_{16} \mathrm{H}_{21} \mathrm{NO}_{2}:$ C, 74.10 (73.89); H, 8.16 (8.01); N, 5.40 (5.37).

2,2-di-(2,2-Dimethyl-propionyloxymethyl)-4,9-dimethyl-1,3,4,9-tetrahydrocarbazole $\quad$ (S17;

Table 2, entry 6). White solid. mp 141-145 ${ }^{\circ} \mathrm{C}$. TLC: $R_{\mathrm{f}}=0.81 .{ }^{1} \mathrm{H}$ NMR (Figure S24): $\delta 7.65$ (d, $J$ $=7.6 \mathrm{~Hz}, 1 \mathrm{H}), 7.65(\mathrm{~d}, J=8.4 \mathrm{~Hz}, 1 \mathrm{H}), 7.18(\mathrm{dt}, J=1.2,7.6 \mathrm{~Hz}, 1 \mathrm{H}), 7.09(\mathrm{td}, J=1.2,7.6 \mathrm{~Hz}, 1 \mathrm{H})$, [4.13 (Abq, $J=10.8 \mathrm{~Hz}), 4.06$ (Abq, $J=11.2 \mathrm{~Hz}), 4 \mathrm{H}], 3.62(\mathrm{~s}, 3 \mathrm{H}), 3.11-3.04(\mathrm{~m}, 1 \mathrm{H}), 2.67$ (Abq, $J$ $=16.8 \mathrm{~Hz}, 2 \mathrm{H}), 2.06(\mathrm{dd}, J=5.2,13.2 \mathrm{~Hz}),[1.48(\mathrm{~d}, J=6.8 \mathrm{~Hz}), 1.46(\mathrm{dd}, J=10.8,13.2 \mathrm{~Hz}), 4 \mathrm{H}]$, 1.27 (s, $9 \mathrm{H}), 1.21(\mathrm{~s}, 9 \mathrm{H}) .{ }^{13} \mathrm{C}\left\{{ }^{1} \mathrm{H}\right\}$ NMR (Figure S25): $\delta$ 178.5, 178.2, 137.8, 132.8, 126.6, 121.0, 
$119.8,119.1,112.9,109.1,68.9,64.7,39.3,39.2,38.8,38.3,29.4,27.6,27.5,25.5,21.8$. IR (neat, $\mathrm{cm}^{-}$ $\left.{ }^{1}\right): 2971,1731,1470,1284,1167,1034$. Anal. Calcd (found) for $\mathrm{C}_{16} \mathrm{H}_{21} \mathrm{NO}_{2}: \mathrm{C}, 73.03$ (73.19); H, 8.72 (8.81); N, $3.28(3.43)$.

\section{2,2-Dicarbo-t-butoxy-4,9-dimethyl-1,3,4,9-tetrahydrocarbazole (S18; Table 2, entry 7).} White solid. mp 140-143 ${ }^{\circ} \mathrm{C}$. TLC: $R_{\mathrm{f}}=0.48 .{ }^{1} \mathrm{H}$ NMR (Figure S26): $\delta 7.62(\mathrm{~d}, J=8.0 \mathrm{~Hz}, 1 \mathrm{H}), 7.28$ (d, $J=8.4 \mathrm{~Hz}, 1 \mathrm{H}), 7.17$ (t, $J=8.0 \mathrm{~Hz}, 1 \mathrm{H}), 7.07$ (t, $J=7.6 \mathrm{~Hz}, 1 \mathrm{H}), 3.68(\mathrm{~s}, 3 \mathrm{H}), 3.41(\mathrm{~d}, J=16.0$ $\mathrm{Hz}, 1 \mathrm{H}), 3.20-3.18(\mathrm{~m}, 1 \mathrm{H}), 2.94(\mathrm{~d}, J=16.0 \mathrm{~Hz}, 1 \mathrm{H}), 2.61(\mathrm{dd}, J=5.6,13.4 \mathrm{~Hz}, 1 \mathrm{H}), 1.82(\mathrm{dd}, J=$ 10.8, $13.0 \mathrm{~Hz}, 1 \mathrm{H}), 1.53$ (s, $9 \mathrm{H}), 1.49$ (d, , $J=6.8 \mathrm{~Hz}, 3 \mathrm{H}), 1.39$ (s, $9 \mathrm{H}) .{ }^{13} \mathrm{C}\left\{{ }^{1} \mathrm{H}\right\}$ NMR (Figure S27): $\delta 171.5,169.8,137.8,133.2,126.7,120.7,119.7,118.8,112.9,109.0,82.1,81.5,55.8,38.8,29.5,28.3$, 28.2, 28.1, 26.4, 21.7. IR (neat, $\mathrm{cm}^{-1}$ ): 2975, 1728, 1470, 1368, 1255, 1167. Anal. Calcd (found) for $\mathrm{C}_{23} \mathrm{H}_{33} \mathrm{NO}_{4}$ : C, 72.15 (71.97); H, 8.33 (8.45); N, 3.51 (3.67).

2,2,4,9-tetramethyl-1,3,4,9-tetrahydrocarbazole (S19; Table 2, entry 8). Colorless oil. TLC (hexanes-EtOAc $=5: 1): R_{\mathrm{f}}=0.64 .{ }^{1} \mathrm{H}$ NMR (Figure S28): $\delta 7.73(\mathrm{~d}, J=7.6 \mathrm{~Hz}, 1 \mathrm{H}), 7.32(\mathrm{~d}, J=7.6$ $\mathrm{Hz}, 1 \mathrm{H}), 7.21(\mathrm{dt}, J=0.8,7.2 \mathrm{~Hz}, 1 \mathrm{H}), 7.13(\mathrm{dt}, J=1.2,7.2 \mathrm{~Hz}, 1 \mathrm{H}), 3.65(\mathrm{~s}, 3 \mathrm{H}), 3.16-3.06(\mathrm{~m}, 1 \mathrm{H})$, $2.53(\mathrm{ABq}, J=16.0 \mathrm{~Hz}, 2 \mathrm{H}), 1.76(\mathrm{dd}, J=5.6,13.0 \mathrm{~Hz}, 1 \mathrm{H}), 1.51(\mathrm{~d}, J=6.8 \mathrm{~Hz}, 3 \mathrm{H}), 1.36(\mathrm{t}, J=7.6$ $\mathrm{Hz}, 1 \mathrm{H}), 1.36(\mathrm{dd}, J=11.2,12.8 \mathrm{~Hz}, 1 \mathrm{H}), 1.23(\mathrm{~s}, 3 \mathrm{H}), 1.02(\mathrm{~s}, 3 \mathrm{H}) .{ }^{13} \mathrm{C}\left\{{ }^{1} \mathrm{H}\right\}$ NMR (Figure S29): $\delta$ $137.7,135.7,127.0,120.4,119.8,118.8,112.7,109.0,47.6,36.5,32.2,31.4,29.3,26.6,25.8,21.7$. IR (neat, $\mathrm{cm}^{-1}$ ): 2950, 2899, 1469, 1371, 1182. Anal. Calcd (found) for $\mathrm{C}_{16} \mathrm{H}_{21} \mathrm{~N}: \mathrm{C}, 84.53$ (84.45); H, 9.31 (9.09); N, $6.16(6.13)$.

\section{7,7-Dicarbomethoxy-5,10-dimethyl-5,6,7,8,9,10-hexahydro-cyclohepta[b]}

indole (S20; Table 2, entry 11). Pale yellow solid. mp 74-76 ${ }^{\circ} \mathrm{C}$. TLC: $R_{\mathrm{f}}=0.47 .{ }^{1} \mathrm{H}$ NMR (Figure S30): $\delta 7.51(\mathrm{~d}, J=7.6 \mathrm{~Hz}, 1 \mathrm{H}), 7.26(\mathrm{~d}, J=8.0 \mathrm{~Hz}, 1 \mathrm{H}), 7.16(\mathrm{dt}, J=1.2,7.2 \mathrm{~Hz}, 1 \mathrm{H}), 7.07(\mathrm{dt}, J=$ 0.8, $7.6 \mathrm{~Hz}, 1 \mathrm{H}), 3.79(\mathrm{~s}, 3 \mathrm{H}), 3.76(\mathrm{~s}, 3 \mathrm{H}), 3.61(\mathrm{~s}, 3 \mathrm{H}), 3.57$ (d, $J=15.6 \mathrm{~Hz}, 1 \mathrm{H}), 3.38-3.32(\mathrm{~m}$, 
with a doublet $\delta 3.35 J=15.6 \mathrm{~Hz}, 2 \mathrm{H}), 2.50(\mathrm{ddd}, J=2.0,10.8,12.0 \mathrm{~Hz}, 1 \mathrm{H}), 2.31-2.25(\mathrm{~m}, 1 \mathrm{H})$, 2.06-1.98 (m, $1 \mathrm{H}), \quad 1.92-1.84(\mathrm{~m}, 1 \mathrm{H}), 1.33(\mathrm{~d}, J=6.8 \mathrm{~Hz}, 3 \mathrm{H}) .{ }^{13} \mathrm{C}\left\{{ }^{1} \mathrm{H}\right\}$ NMR (Figure S31): $\delta$ $172.9,171.4,136.7,132.0,127.2,121.0,118.9,118.2,117.5,109.5,57.2,53.2,52.8,31.0,30.2,29.9$, 29.6, 29.5, 20.3. Anal. Calcd (found) for $\mathrm{C}_{19} \mathrm{H}_{23} \mathrm{NO}_{4}$ : C, 69.28 (69.17); H, 7.04 (6.92); N, 4.25 (4.24).

Cyclization of 7 Catalyzed by [(S)-4]PtCl$/ 2 / \mathbf{A g O T f}$. A suspension of 7 (63 $\mathrm{mg}, 0.23 \mathrm{mmol})$, $[(S)-4] \mathrm{PtCl}_{2}$ (33mg, $\left.0.023 \mathrm{mmol}\right)$, and $\mathrm{AgOTf}(6 \mathrm{mg}, 0.023)$ in $0.90 \mathrm{ml}$ of $\mathrm{MeOH}$ was stirred at $60{ }^{\circ} \mathrm{C}$ for 64 hours, cooled to room temperature, and filtered through a plug of silica gel, eluting with ether. The filtrate was concentrated under vacuum and the residue was chromatographed (hexanes-EtOAc $=$ 10:1) to give a 9:1 mixture of cis-8 and trans-8 (59 mg 94\%) that contained $\sim 5 \%$ of an unidentified impurity. The enantiomeric excess of cis-8 and trans-8 were determined by HPLC analysis of the corresponding alcohols cis- and trans-2-methylhydroxy-4,9-dimethyl-1,3,4,9-tetrahydrocarbazole (S21), generated via $\mathrm{LiAlH}_{4}$ reduction of cis-8 and trans-8 (Figure S13).

Cyclization of 7 Catalyzed by $\left[( \pm)-\mathbf{B I N A P}_{\mathbf{P}} \mathbf{P t C l}_{2}\right.$. A suspension of $7(69 \mathrm{mg}, 0.25 \mathrm{mmol}),[( \pm)-$ BINAP]PtCl,$(22 \mathrm{mg}, 0.025 \mathrm{mmol})$, and $\mathrm{AgOTf}(6 \mathrm{mg}, 0.025 \mathrm{mmol})$ in $\mathrm{MeOH}(1 \mathrm{~mL})$ was stirred at 60 ${ }^{\circ} \mathrm{C}$ for 38 hours, cooled to room temperature, and filtered through a plug of silica gel, eluting with ether. The filtrate was concentrated under vacuum and the residue was chromatographed (hexanes-EtOAc $=$ 10:1) to give a $25: 1$ mixture of cis-8 and trans-8 (64 mg, 93\%).

For cis-8: ${ }^{1} \mathrm{H}$ NMR $(500 \mathrm{MHz}$, Figure S32): $\delta 7.66(\mathrm{~d}, J=8.0 \mathrm{~Hz}, 1 \mathrm{H}), 7.30(\mathrm{~d}, J=8.0 \mathrm{~Hz}, 1$ H), $7.20(\mathrm{t}, J=7.6 \mathrm{~Hz}, 1 \mathrm{H}), 7.10(\mathrm{t}, J=8.0 \mathrm{~Hz}, 1 \mathrm{H}), 4.34(\mathrm{dd}, J=5.6,9.6 \mathrm{~Hz}, 1 \mathrm{H}), 4.27(\mathrm{dd}, J=7.2$, $9.6 \mathrm{~Hz}, 1 \mathrm{H}), 3.80(\mathrm{~s}, 3 \mathrm{H}), 3.65$ (s, $3 \mathrm{H}), 3.24-3.14(\mathrm{~m} 1 \mathrm{H}), 3.02$ (tdd, $J=1.5,5.5,16.0 \mathrm{~Hz}, 1 \mathrm{H}), 2.95$ (ddd, $J=2.5,10.5,16.0 \mathrm{~Hz}, 1 \mathrm{H}), 2.90-2.83(\mathrm{~m}, 1 \mathrm{H}), 2.44-2.39(\mathrm{~m}, 1 \mathrm{H}), 1.57(\mathrm{dt} J=11.0,13.0 \mathrm{~Hz}, 1$ $\mathrm{H}), 1.50(\mathrm{~d}, J=6.5 \mathrm{~Hz}, 3 \mathrm{H}) .{ }^{13} \mathrm{C}\left\{{ }^{1} \mathrm{H}\right\}$ NMR (126 MHz, Figure S33): $\delta$ 137.6, 134.0, 126.6, 120.9, $119.8,119.1,109.1,74.1,37.7,36.8,35.2,29.4,29.0,25.8,21.8$. 
Stereochemistry of $c i s-\mathbf{8}$. The stereochemistry of $c i s-\mathbf{8}$ was determined by conversion of $c i s-\mathbf{8}$ to cis-2,4,9-trimethyl-1,3,4,9-tetrahydrocarbazole (cis-S22) in 46\% yield over three steps (Scheme S1), followed by X-ray crystallographic analysis of cis-S22 (Figure S34, Tables S3-S7). The crystal of cisS22 used for X-ray analysis was recovered and subjected to GC analysis, which confirmed its identity as the major diastereomer.
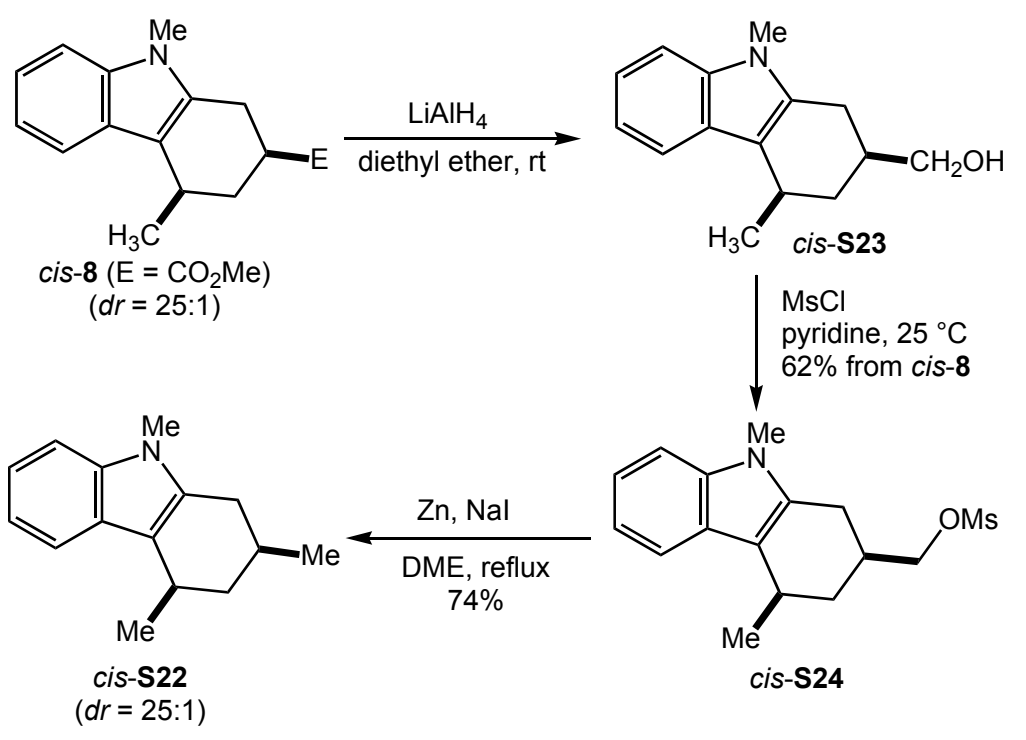

\section{Scheme S1}

cis-2,4,9-trimethyl-1,3,4,9-tetrahydrocarbazole (cis-S22). A solution of $(\mathbf{8}$, cis:trans $=25: 1$, $64 \mathrm{mg}, 0.24 \mathrm{mmol})$ in diethyl ether $(3 \mathrm{~mL})$ was added slowly to a suspension of $\mathrm{LiAlH}_{4}(18 \mathrm{mg}, 0.48$ mmol) in diethyl ether $(2 \mathrm{~mL})$ at room temperature. The reaction mixture was stirred at room temperature for 40 minutes and quenched via slow addition of water. The resulting mixture was filtered through a short plug of silica gel and eluted with ether $(5 \mathrm{~mL})$. The filtrate and eluant were combined and concentrated under vacuum to give crude 2-hydroxymethyl-4,9-dimethyl-1,3,4,9-tetrahydrocarbazole (S23) that was used in the next step without further purification. Methane sulfonylchloride $(0.3 \mathrm{~mL}, 3.9 \mathrm{mmol})$ was added dropwise to a solution of crude $\mathbf{S 2 3}$ in pyridine (2.0 
$\mathrm{mL}$ ) at $0{ }^{\circ} \mathrm{C}$. The reaction mixture was stirred at room temperature for 2 hours, quenched with ice-water $(10 \mathrm{~mL})$, and extracted with ether $(50 \mathrm{~mL})$. The combined ether extracts were washed with $0.5 \mathrm{HCl}(2 \times$ $20 \mathrm{~mL})$, saturated aqueous $\mathrm{NaHCO}_{3}(20 \mathrm{~mL})$, and brine $(20 \mathrm{~mL})$, dried $\left(\mathrm{MgSO}_{4}\right)$, and concentrated under vacuum. The residue was chromatographed (hexanes-EtOAc $=3: 1)$ to give $\mathbf{S 2 4}(46 \mathrm{mg}, 62 \%$ from 8 ) as a pale yellow oil.

A mixture of S24 (44 mg, $0.14 \mathrm{mmol}), \mathrm{NaI}(63 \mathrm{mg}, 0.42 \mathrm{mmol})$, and Zn power (55 mg, 0.84 mmol) in DME $(2.5 \mathrm{~mL})$ and water $(0.05 \mathrm{~mL})$ was refluxed for 3 hours. The resulting mixture was cooled to room temperature, filtered through a short plug of silica gel and eluted with ether $(5 \mathrm{~mL})$. The filtrate and eluant were combined and concentrated under vacuum. The residue was chromatographed (hexanes-diethyl ether $=20: 1)$ to give a $25: 1$ mixture of cis-S22 and trans-S22 (23 $\mathrm{mg}, 74 \%$ yield) as a white solid. Crystals of pure cis-S22 suitable for X-ray analysis were obtained by slow evaporation of a hexanes/ether solution.

For cis-S22: TLC: $R_{\mathrm{f}}=0.68 .{ }^{1} \mathrm{H}$ NMR: $\delta 7.67(\mathrm{~d}, J=8.0 \mathrm{~Hz}, 1 \mathrm{H}), 7.28(\mathrm{~d}, J=8.0 \mathrm{~Hz}, 1 \mathrm{H})$, $7.16(\mathrm{t}, J=7.6 \mathrm{~Hz}, 1 \mathrm{H}), 7.07(\mathrm{t}, J=7.6 \mathrm{~Hz}, 1 \mathrm{H}), 3.63(\mathrm{~s}, 3 \mathrm{H}), 3.16-3.04(\mathrm{~m}, 1 \mathrm{H}), 2.78(\mathrm{~d}, J=3.6 \mathrm{~Hz}$ 16.0, $1 \mathrm{H}), 2.29$ (ddd, $J=2.4,10.8,15.2 \mathrm{~Hz}, 1 \mathrm{H}), 2.40-1.90(\mathrm{~m}, 2 \mathrm{H}), 1.46(\mathrm{~d}, J=6.8 \mathrm{~Hz}, 3 \mathrm{H}), 1.20-$ $1.11(\mathrm{~m}, 4 \mathrm{H}$ with a doublet at $\delta 6.4, J=7.6 \mathrm{~Hz}) .{ }^{13} \mathrm{C}\left\{{ }^{1} \mathrm{H}\right\}$ NMR: $\delta 137.5,136.2,126.9,120.5,119.8$, 118.8, 114.1, 109.0, 43.4, 31.2, 29.6, 29.3, 22.7, 22.0. IR (neat, $\mathrm{cm}^{-1}$ ): 2950, 1470, 1413, 1376, 1241. Anal. Calcd (found) for $\mathrm{C}_{14} \mathrm{H}_{17} \mathrm{~N}$ : C 84.46 (84.34); H 8.98 (9.09); N 6.57 (6.60).

For cis-S24: TLC: $R_{\mathrm{f}}=0.28 .{ }^{1} \mathrm{H}$ NMR: $\delta 7.66(\mathrm{~d}, J=8.0 \mathrm{~Hz}, 1 \mathrm{H}), 7.29(\mathrm{~d}, J=8.4 \mathrm{~Hz}, 1 \mathrm{H})$, $7.19(\mathrm{dt}, J=0.8,8.0 \mathrm{~Hz}, 1 \mathrm{H}), 7.09(\mathrm{dt}, J=0.8,8.0 \mathrm{~Hz}, 1 \mathrm{H}), 4.34(\mathrm{dd}, J=5.6,9.6 \mathrm{~Hz}, 1 \mathrm{H}), 4.27(\mathrm{dd}, J$ $=7.2,9.6 \mathrm{~Hz}, 1 \mathrm{H}), 3.64(\mathrm{~s}, 3 \mathrm{H}), 3.20-3.10(\mathrm{~m} 1 \mathrm{H}), 3.08(\mathrm{~s}, 3 \mathrm{H}), 2.92(\mathrm{dd}, J=5.2,15.8 \mathrm{~Hz}, 1 \mathrm{H}), 2.50$ (ddd, $J=2.4,11.2,15.8 \mathrm{~Hz}, 1 \mathrm{H}), 2.40-2.28(\mathrm{~m}, 1 \mathrm{H}), 2.12-2.07(\mathrm{~m}, 1 \mathrm{H}), 1.48(\mathrm{~d}, J=6.8 \mathrm{~Hz}, 3 \mathrm{H}), 1.26$ $(\mathrm{q}, J=12.4 \mathrm{~Hz}, 1 \mathrm{H}) .{ }^{13} \mathrm{C}\left\{{ }^{1} \mathrm{H}\right\}$ NMR: $\delta 137.6,134.0,126.6,120.9,119.8,119.1,109.1,74.1,37.7$, 
36.8, 35.2, 29.4, 29.0, 25.8, 21.8. IR (neat, $\mathrm{cm}^{-1}$ ): 2920, 1470, 1353, 1174, 955, 832. HRMS calcd (found) for $\mathrm{C}_{16} \mathrm{H}_{21} \mathrm{NO}_{3} \mathrm{~S}: 307.1242$ (307.1241). 
Figure S1. Chiral HPLC trace for racemic 5 (left trace) and enantiomerically enriched 5 (right trace) (hexanes/isopropanol $=90 / 10,0.6 \mathrm{~mL} / \mathrm{min}$ ).

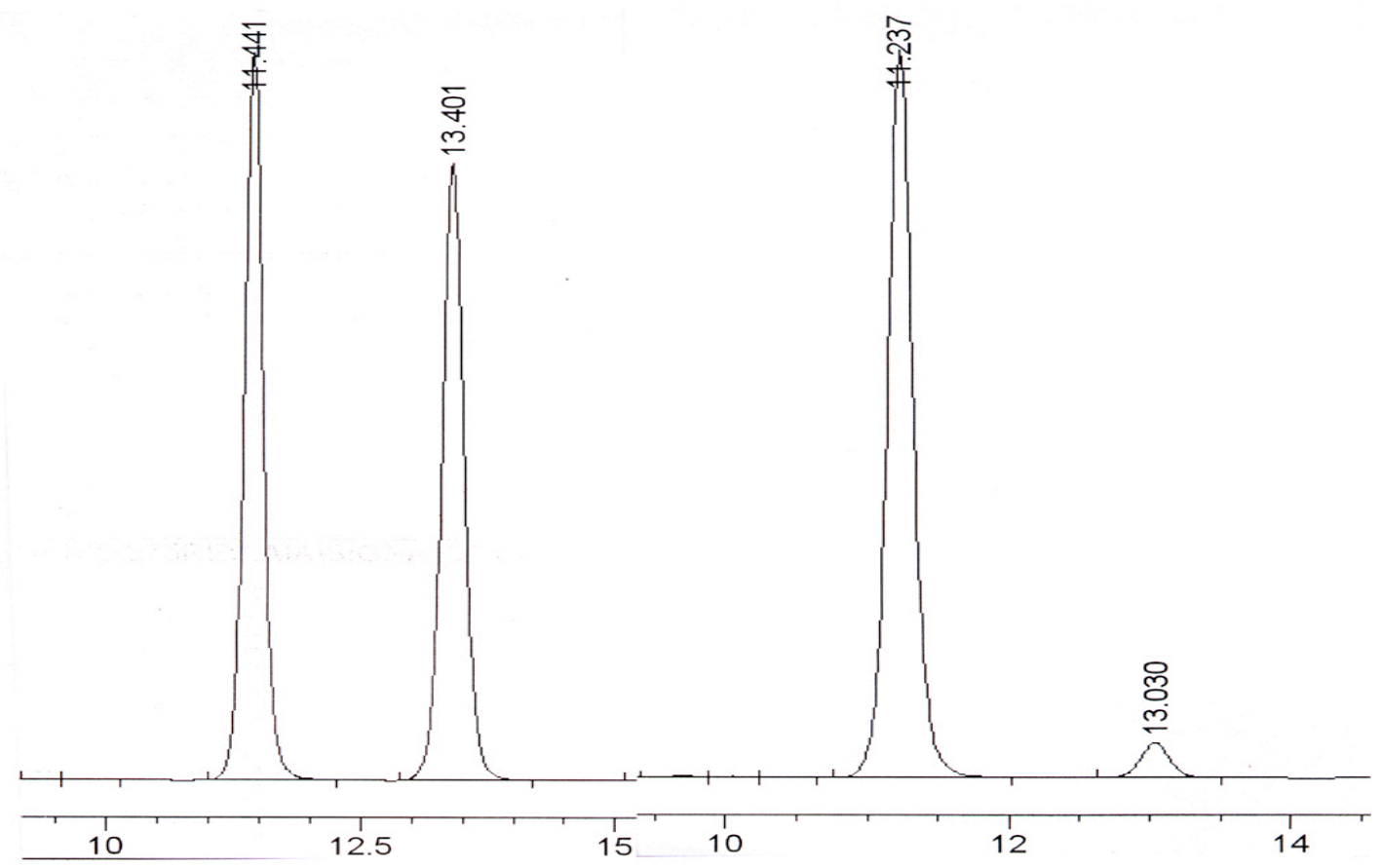

Figure S2. Chiral HPLC trace for racemic S12 (left trace) and enantiomerically enriched S12 (right trace) (hexanes/isopropanol $=90 / 10,0.5 \mathrm{~mL} / \mathrm{min})$.
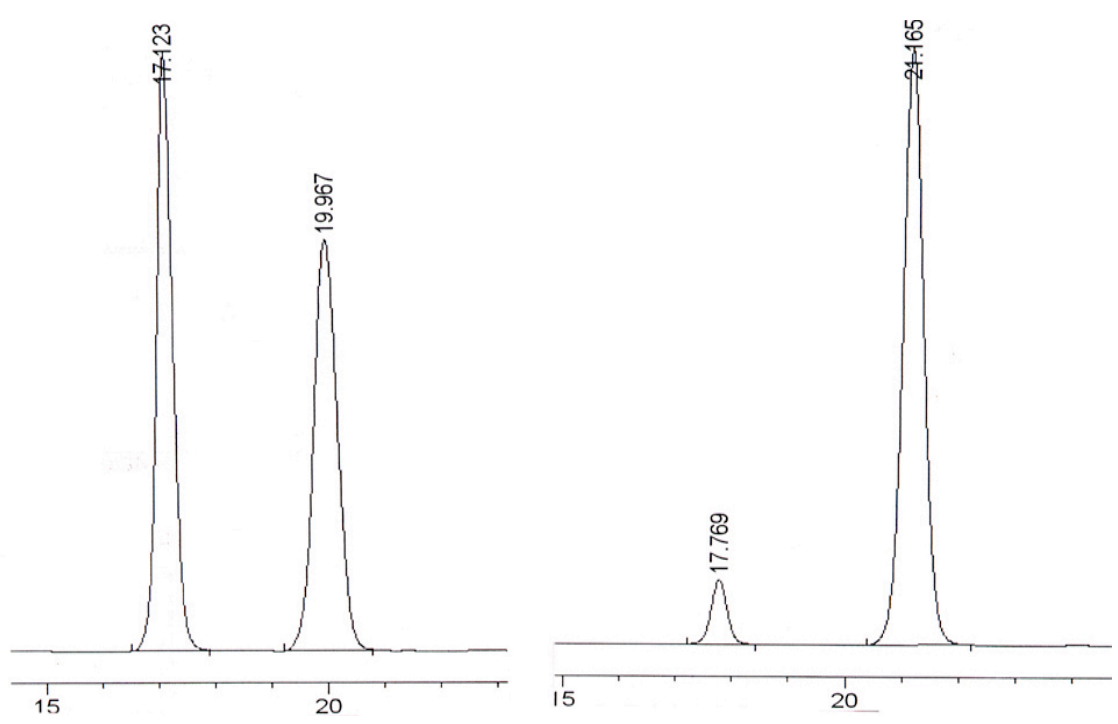
Figure S3. Chiral HPLC trace for racemic S13 (left trace) and enantiomerically enriched S13 (right trace) (hexanes/isopropanol $=90 / 10,0.35 \mathrm{~mL} / \mathrm{min})$.
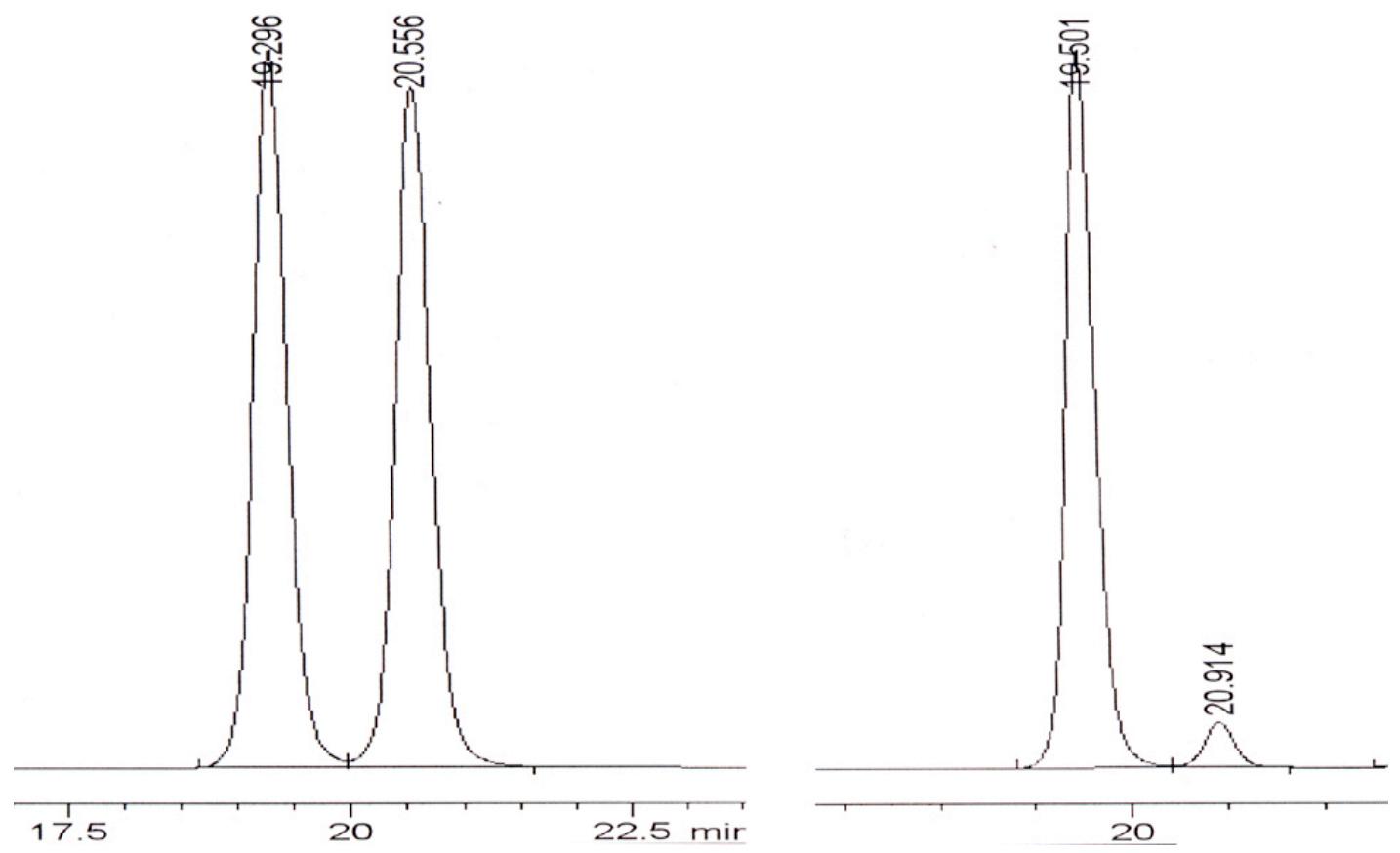

Figure S4. Chiral HPLC trace for racemic S14 (left trace) and enantiomerically enriched S14 (right trace) (hexanes/isopropanol $=80 / 20,0.5 \mathrm{~mL} / \mathrm{min})$.
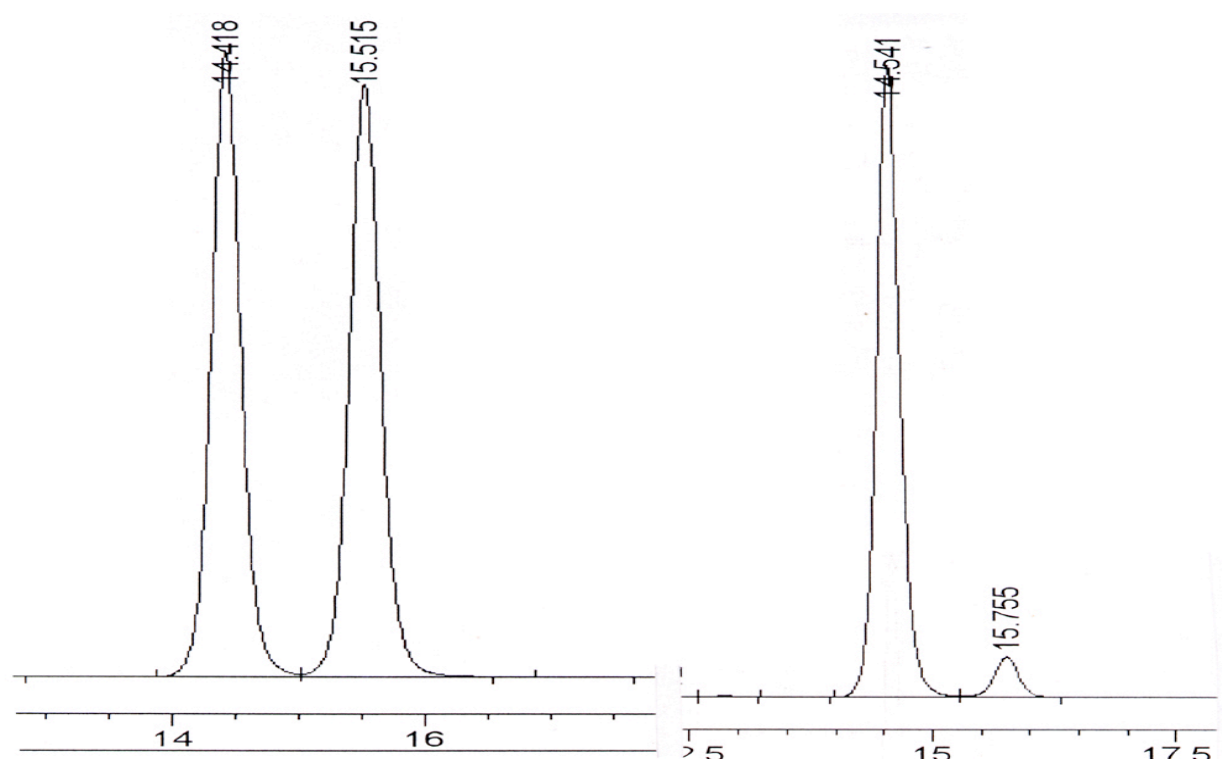
Figure S5. Chiral HPLC trace for racemic S15 (left trace) and enantiomerically enriched S15 (right trace) (hexanes/isopropanol $=80 / 20,0.5 \mathrm{~mL} / \mathrm{min})$.
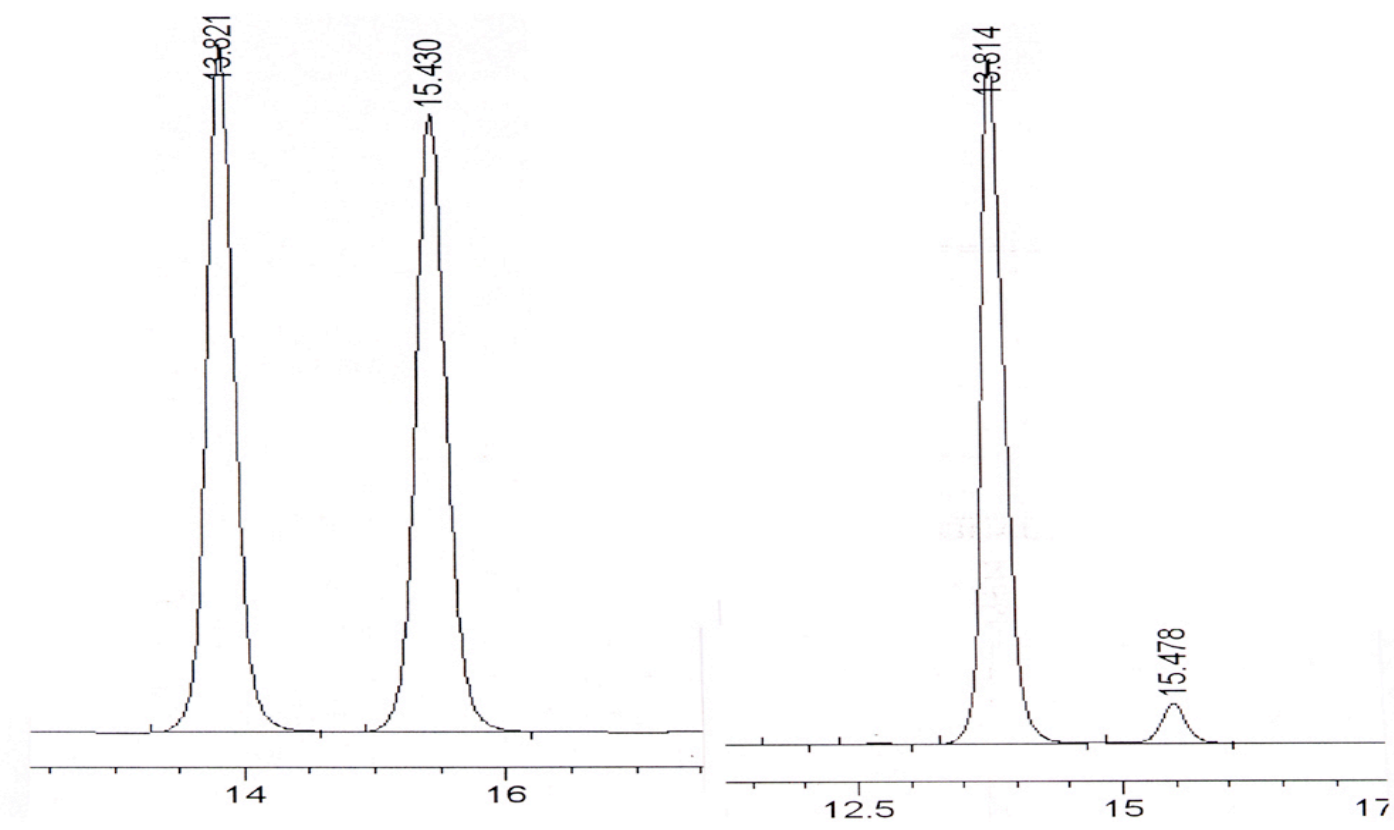

Figure S6. Chiral HPLC trace for racemic S16 (left trace) and enantiomerically enriched S16 (right trace) (hexanes/isopropanol $=85 / 15,0.5 \mathrm{~mL} / \mathrm{min})$.
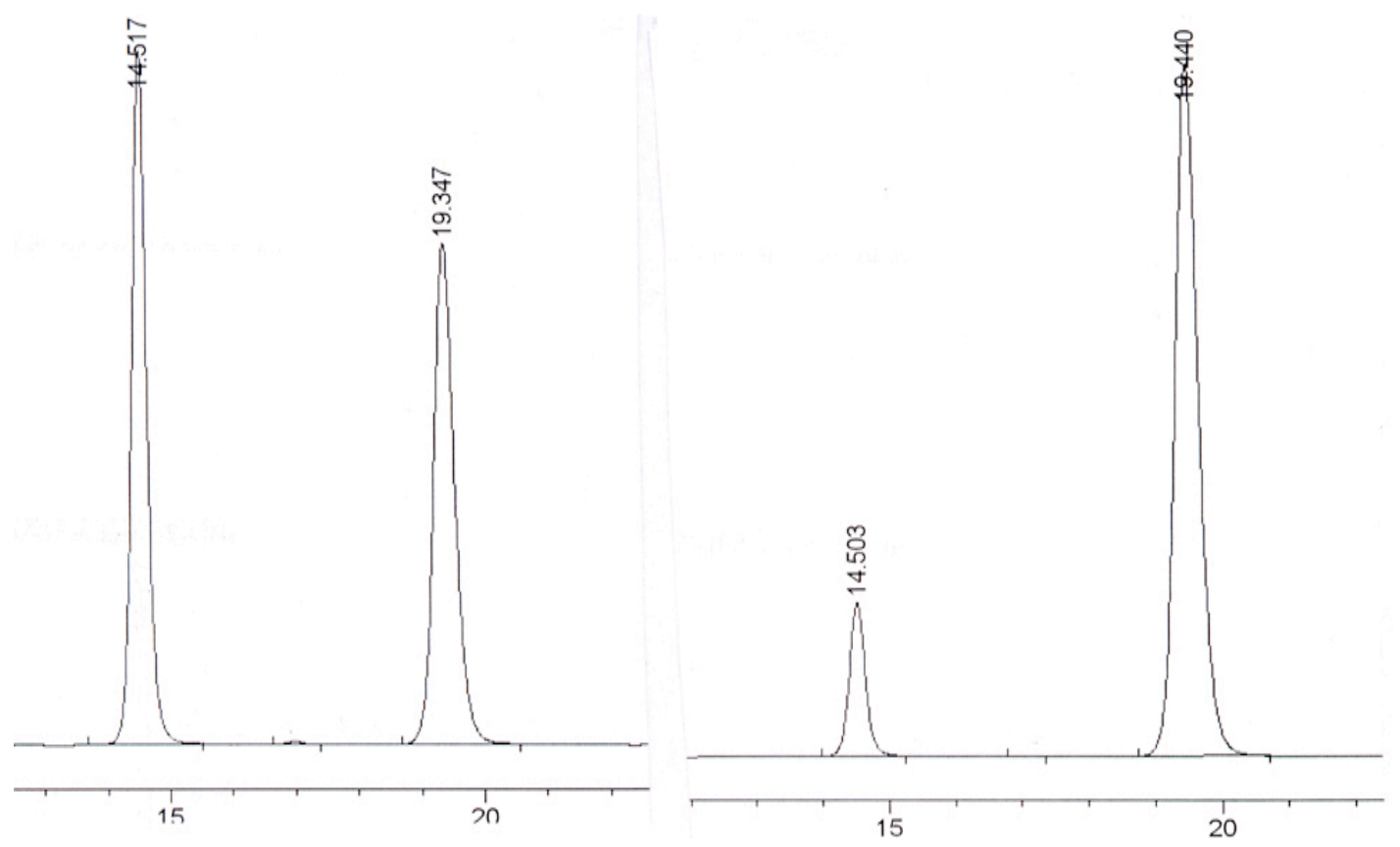
Figure S7. Chiral HPLC trace for racemic S17 (left trace) and enantiomerically enriched S17 (right trace) $($ hexanes $/$ isopropanol $=99.5 / 0.5,0.5 \mathrm{~mL} / \mathrm{min})$.
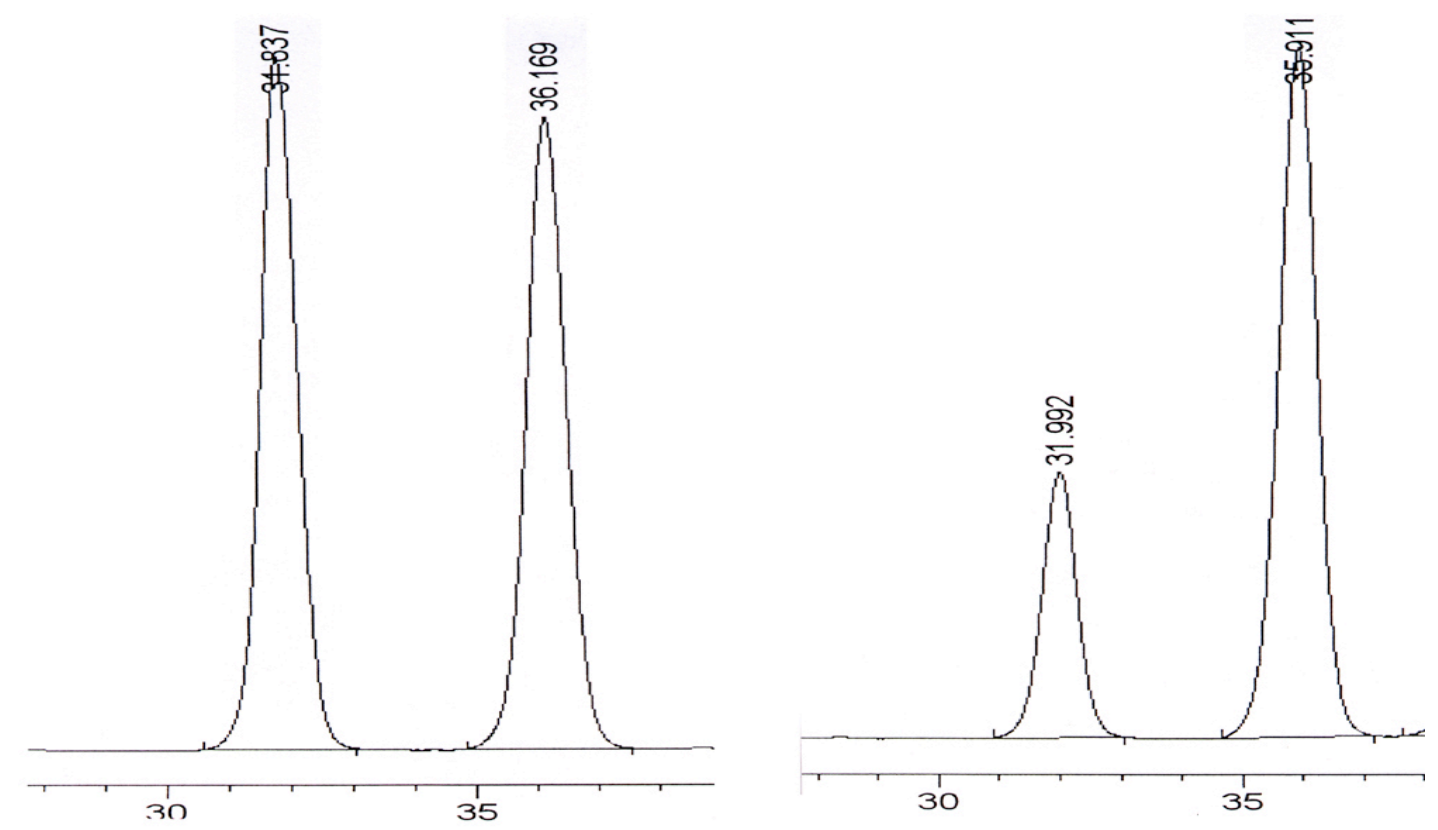

Figure S8. Chiral HPLC trace for racemic $\mathbf{S 1 6}$ (left trace) and $\mathbf{S 1 6}$ synthesized via $\mathrm{LiAH}_{4}$ reduction of enantiomerically enriched $\mathbf{S 1 8}$ (right trace, hexanes/isopropanol $=85 / 15,0.5 \mathrm{~mL} / \mathrm{min}$ ).
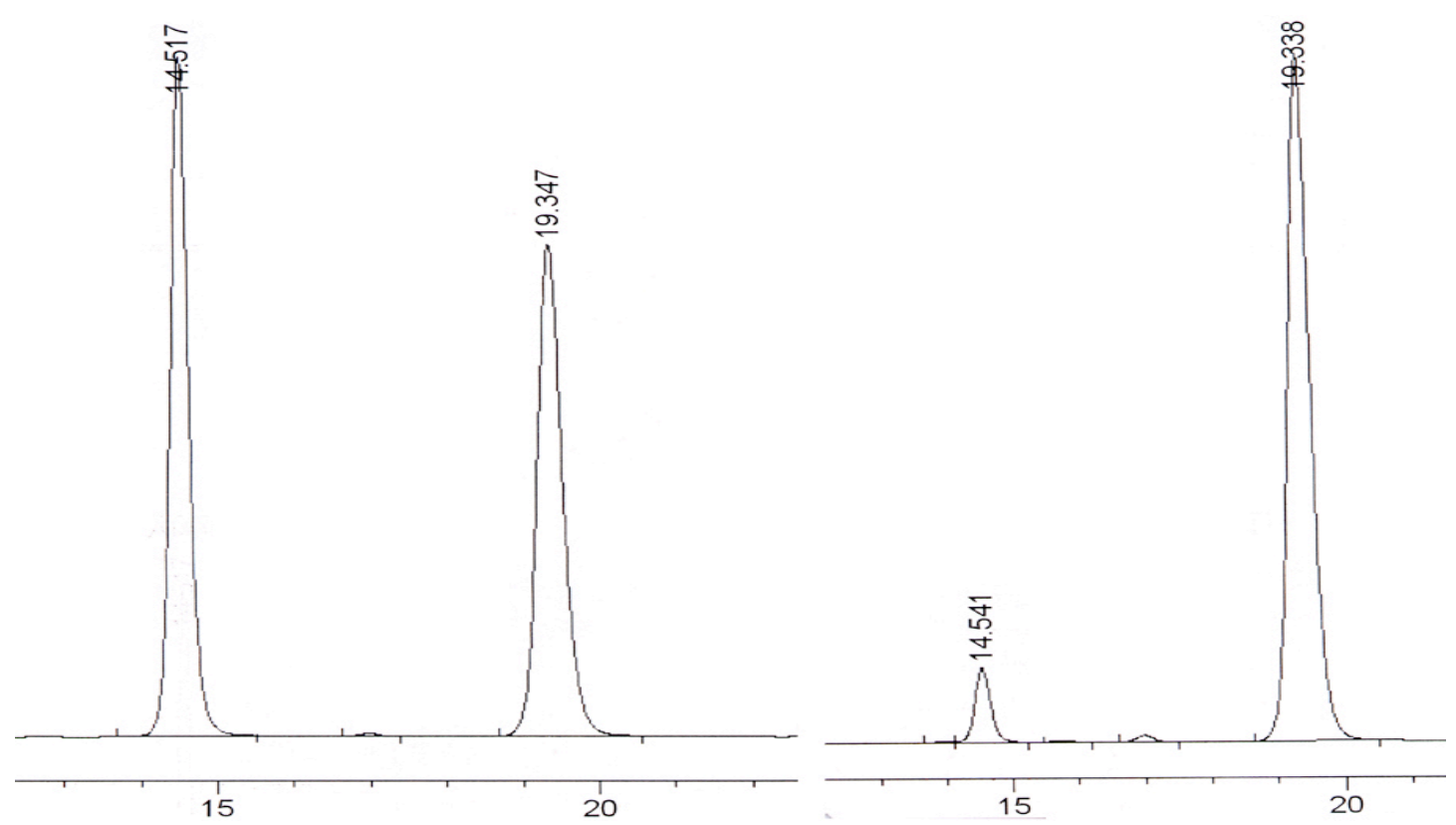
Figure S9. Chiral HPLC trace for racemic S19 (left trace) and enantiomerically enriched S19 (right trace) $($ hexanes/isopropanol $=99.5 / 0.5,0.5 \mathrm{~mL} / \mathrm{min})$.
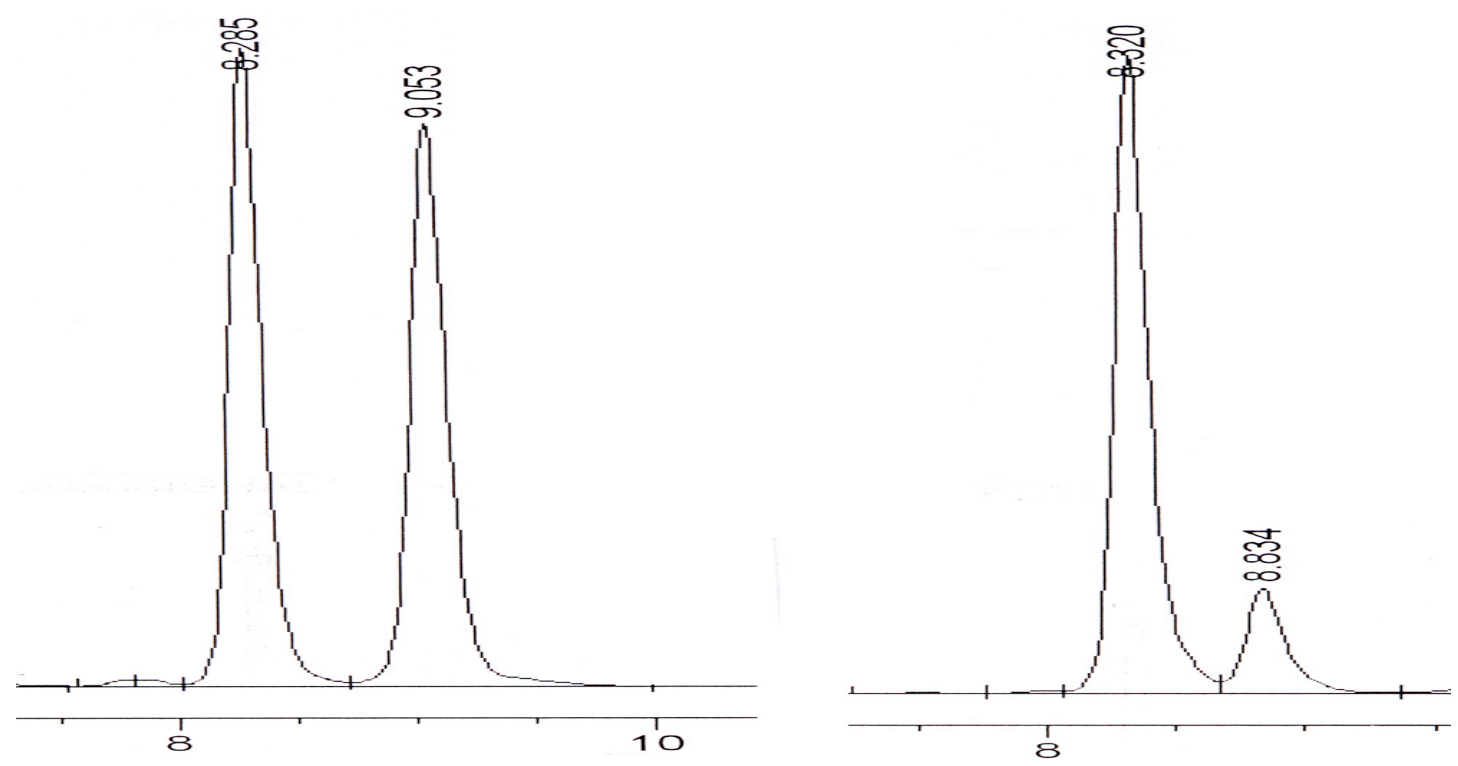

Figure S10. Chiral HPLC trace for racemic 1,1,4,9-tetramethyl-2,3,4,9-tetrahydrocarbazole (left trace) and enantiomerically enriched 1,1,4,9-tetramethyl-2,3,4,9-tetrahydrocarbazole (right trace) (hexanes/isopropanol $=99.7 / 0.3,0.5 \mathrm{~mL} / \mathrm{min}$ ).
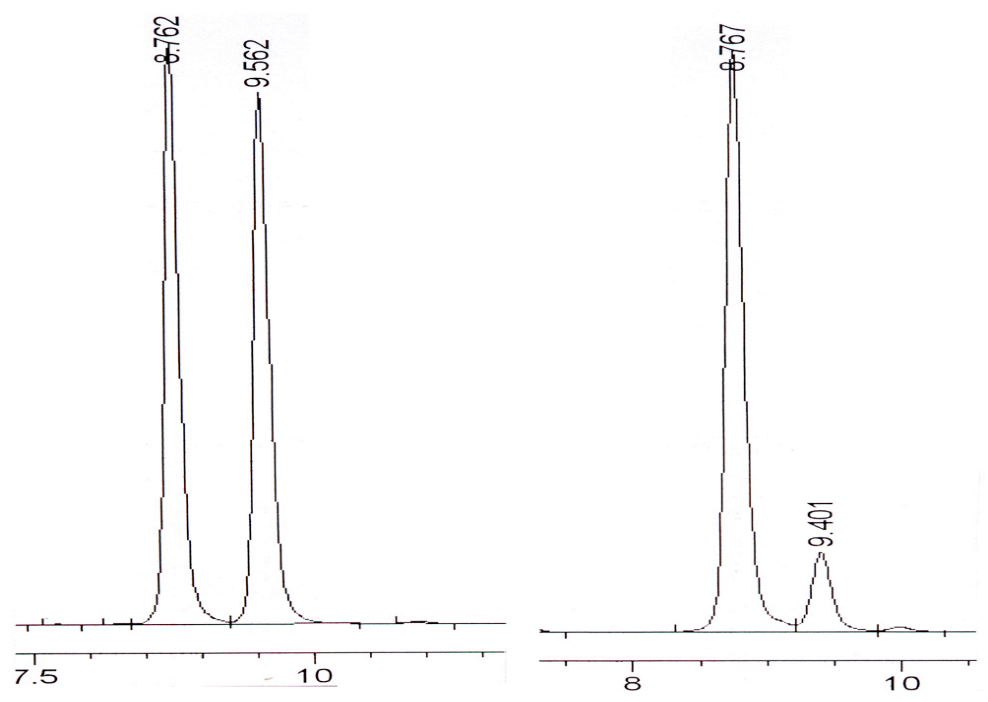
Figure S11. Chiral HPLC trace for racemic 1,1,4-trimethyl-2,3,4,9-tetrahydrocarbazole (left trace) and enantiomerically enriched 1,1,4-trimethyl-2,3,4,9-tetrahydrocarbazole (right trace) (hexanes/isopropanol $=99 / 1,0.5 \mathrm{~mL} / \mathrm{min})$.
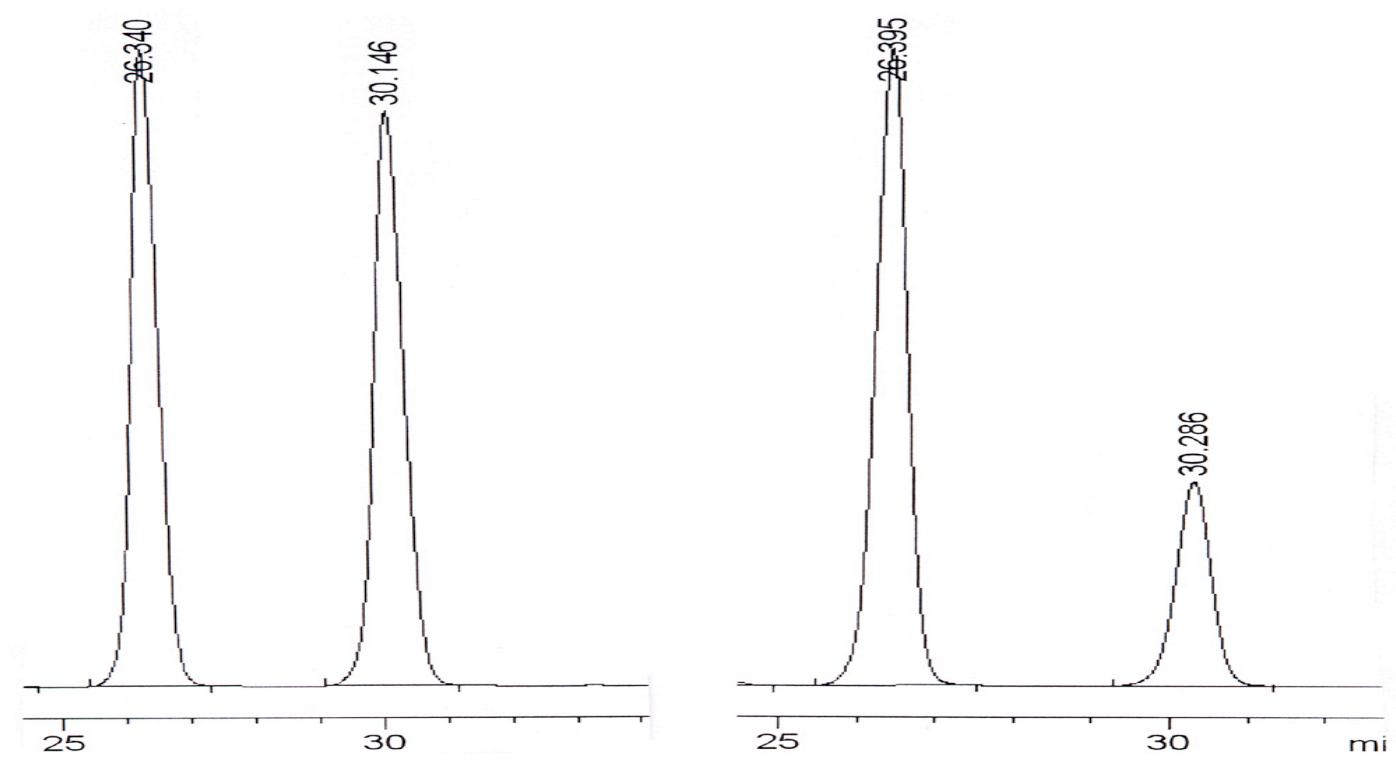

Figure S12. Chiral HPLC trace for racemic mixture of S20 (left trace) and enantiomerically enriched S20 (right trace) (hexanes/isopropanol $=98 / 2,0.5 \mathrm{~mL} / \mathrm{min}$ ).
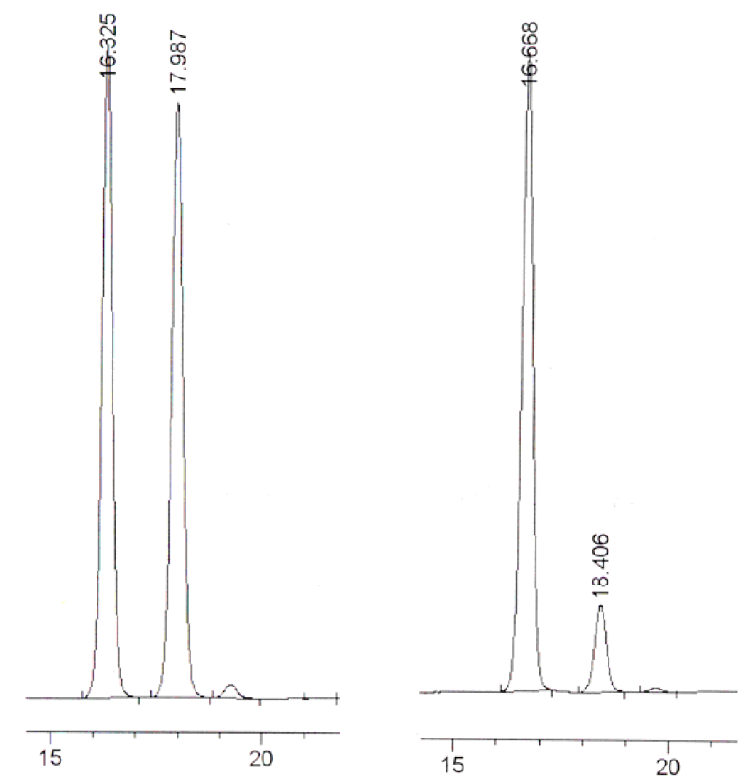
Figure S13. Chiral HPLC trace for racemic mixture of S21 (cis:trans = 1:2) (left trace) and enantiomerically enriched S21 (cis:trans = 9:1) (right trace) (hexanes/isopropanol = 98.5/1.5, 0.3 $\mathrm{mL} / \mathrm{min})$.

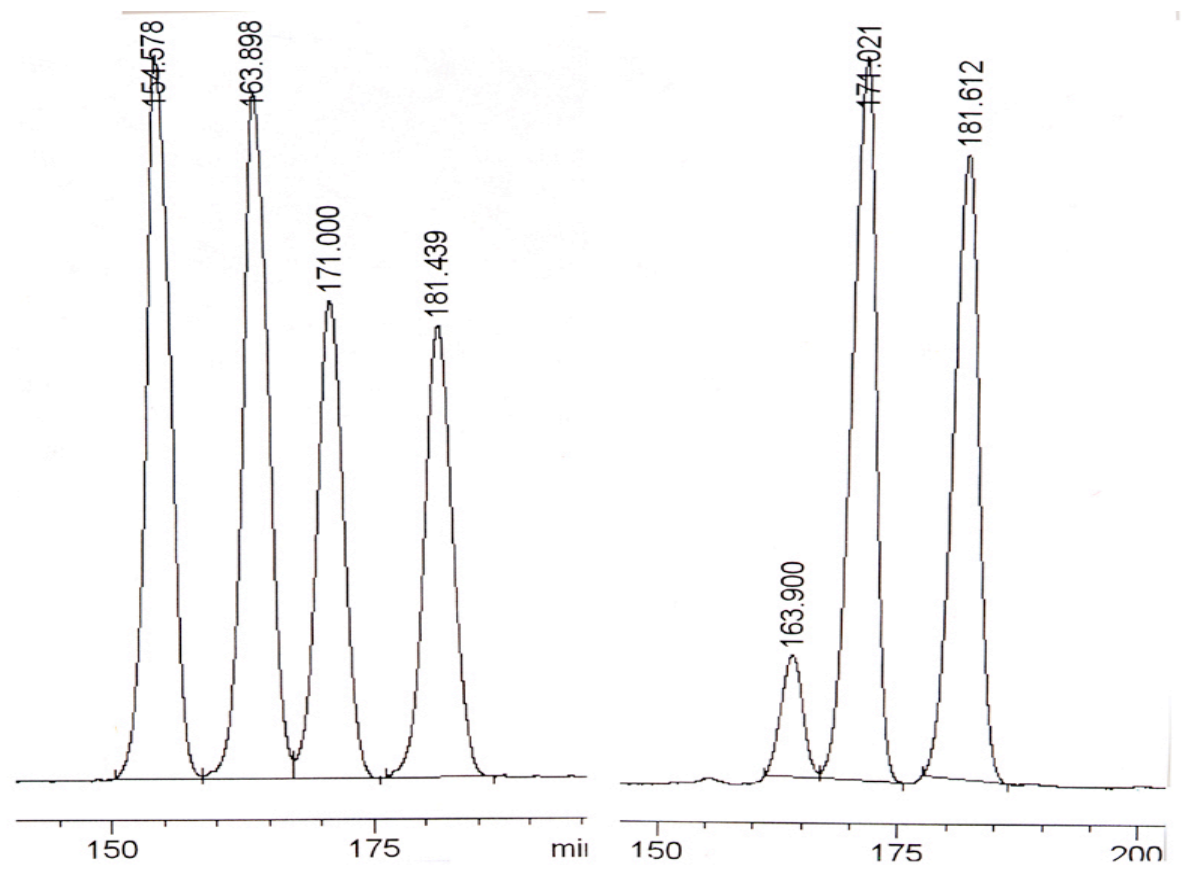


Figure S14. ${ }^{1} \mathrm{H}$ NMR spectrum of $\mathbf{S 1 2}$ in $\mathrm{CDCl}_{3}$.
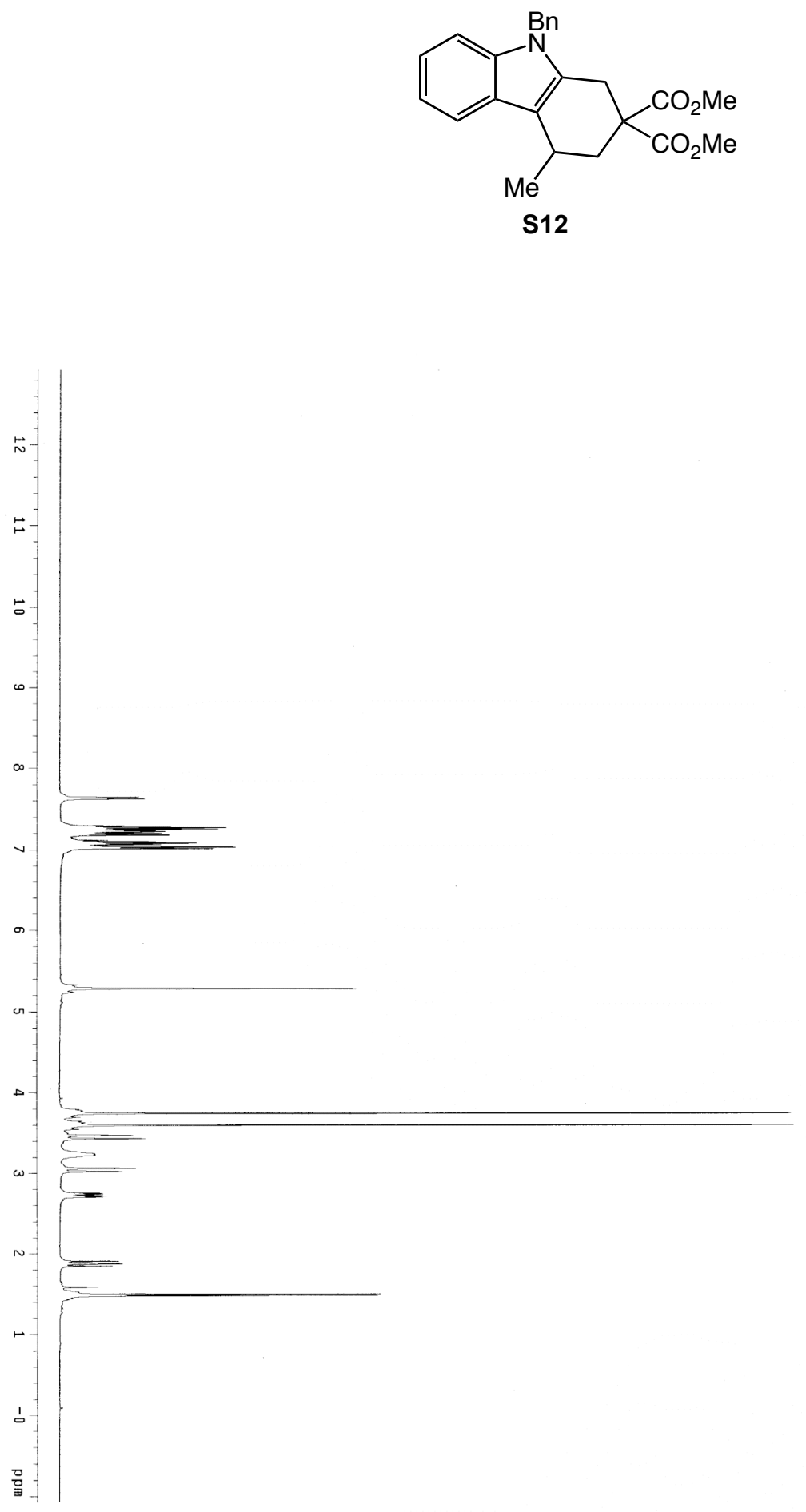
Figure S15. ${ }^{13} \mathrm{C}\left\{{ }^{1} \mathrm{H}\right\}$ spectrum of $\mathbf{S 1 2}$ in $\mathrm{CDCl}_{3}$.<smiles>CC(=O)C1(C(C)=O)Cc2[nH]c3ccccc3c2C(C)C1</smiles>

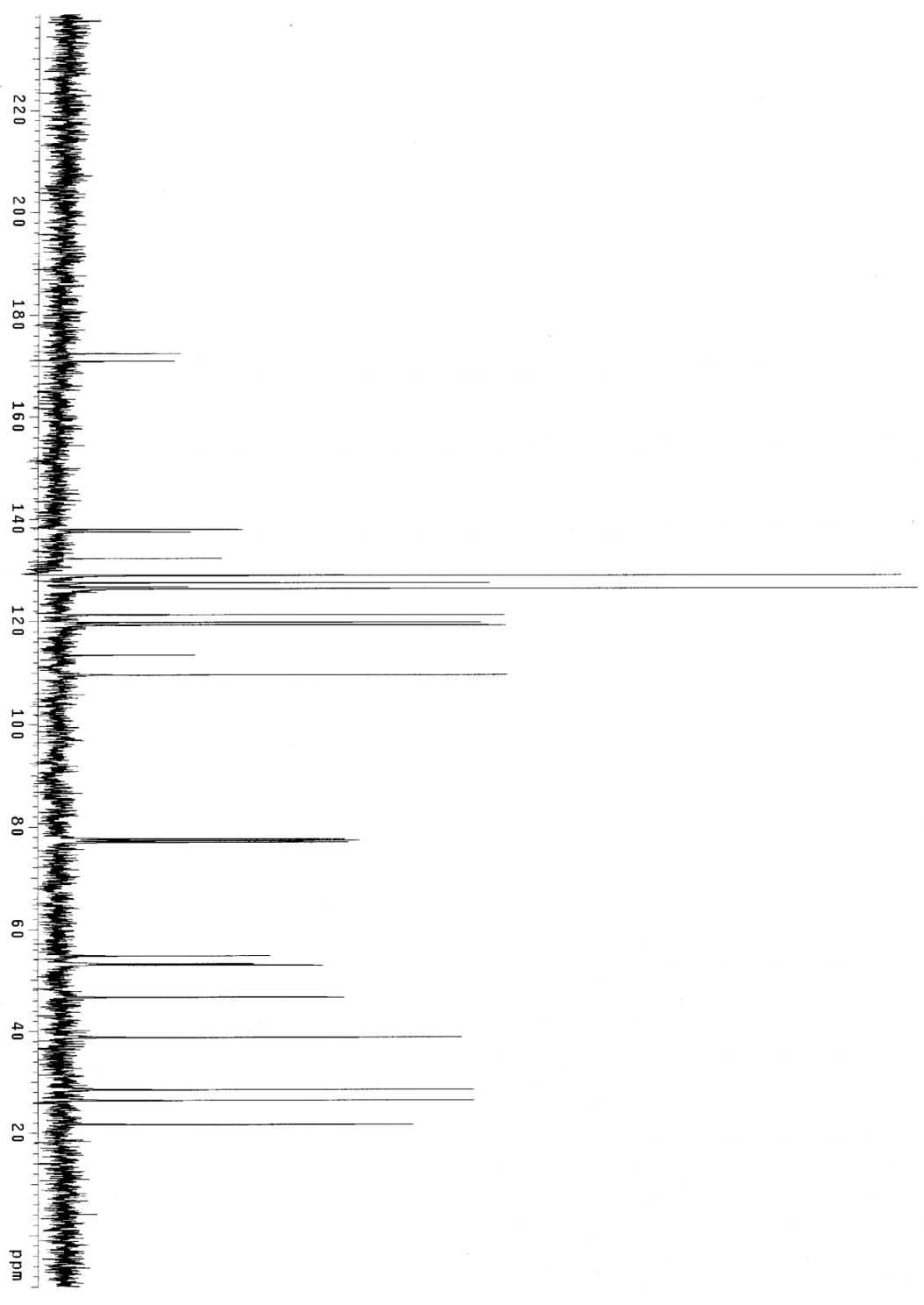


Figure S16. ${ }^{1} \mathrm{H}$ NMR spectrum of $\mathbf{S 1 3}$ in $\mathrm{CDCl}_{3}$.
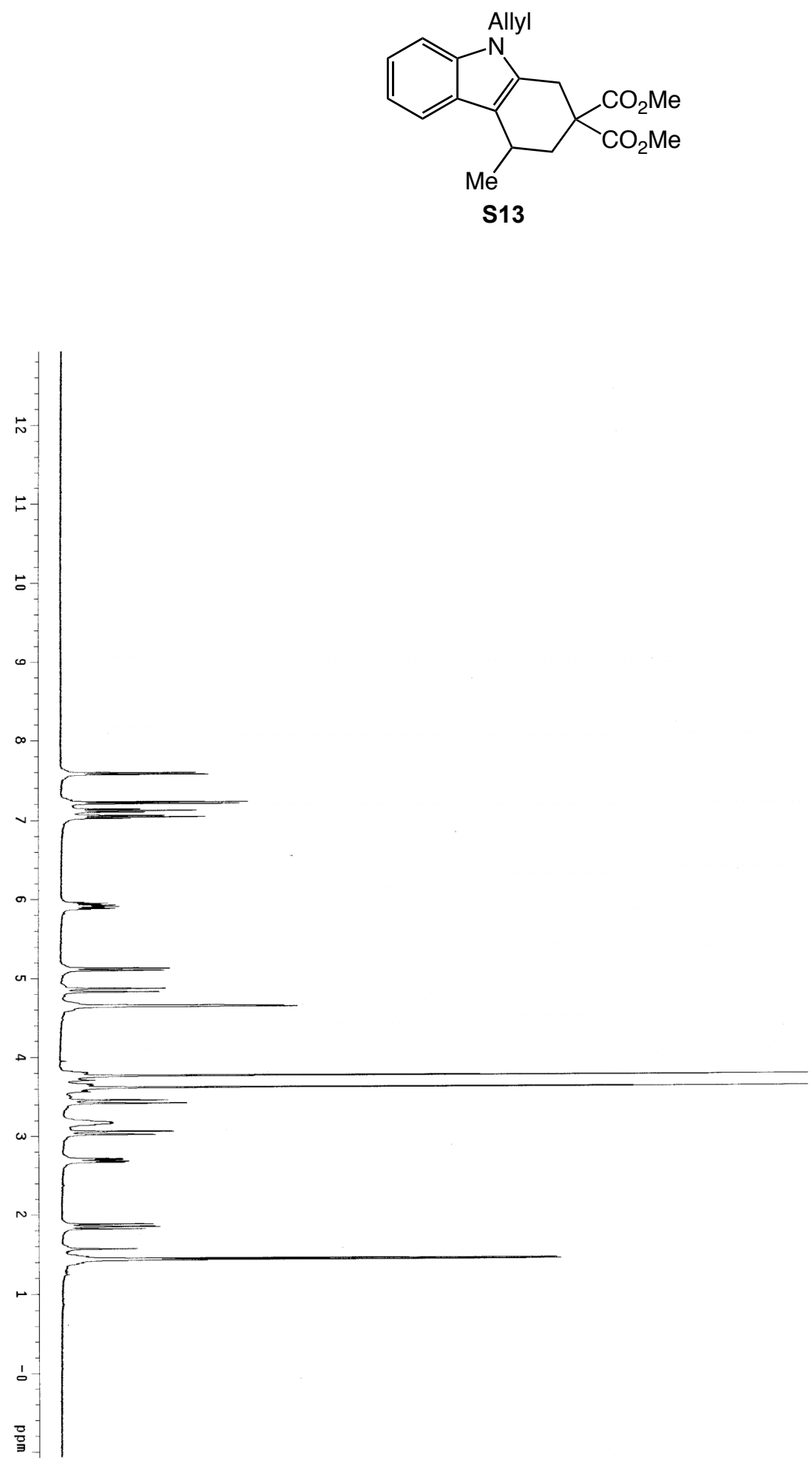


$$
E
$$


Figure S18. ${ }^{1} \mathrm{H}$ spectrum of $\mathbf{S 1 4}$ in $\mathrm{CDCl}_{3}$
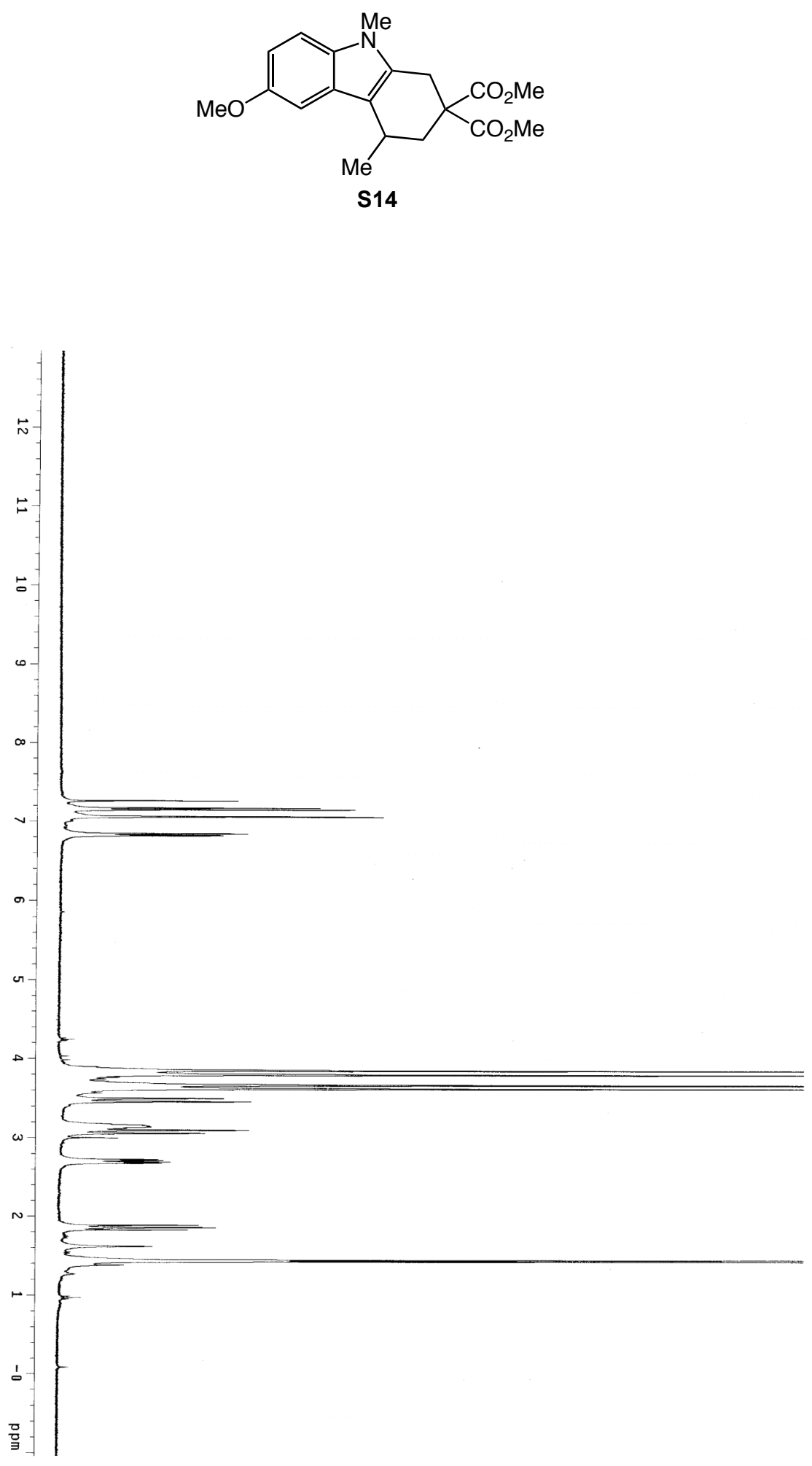
Figure S19. ${ }^{13} \mathrm{C}\left\{{ }^{1} \mathrm{H}\right\}$ spectrum of $\mathbf{S 1 4}$ in $\mathrm{CDCl}_{3}$.
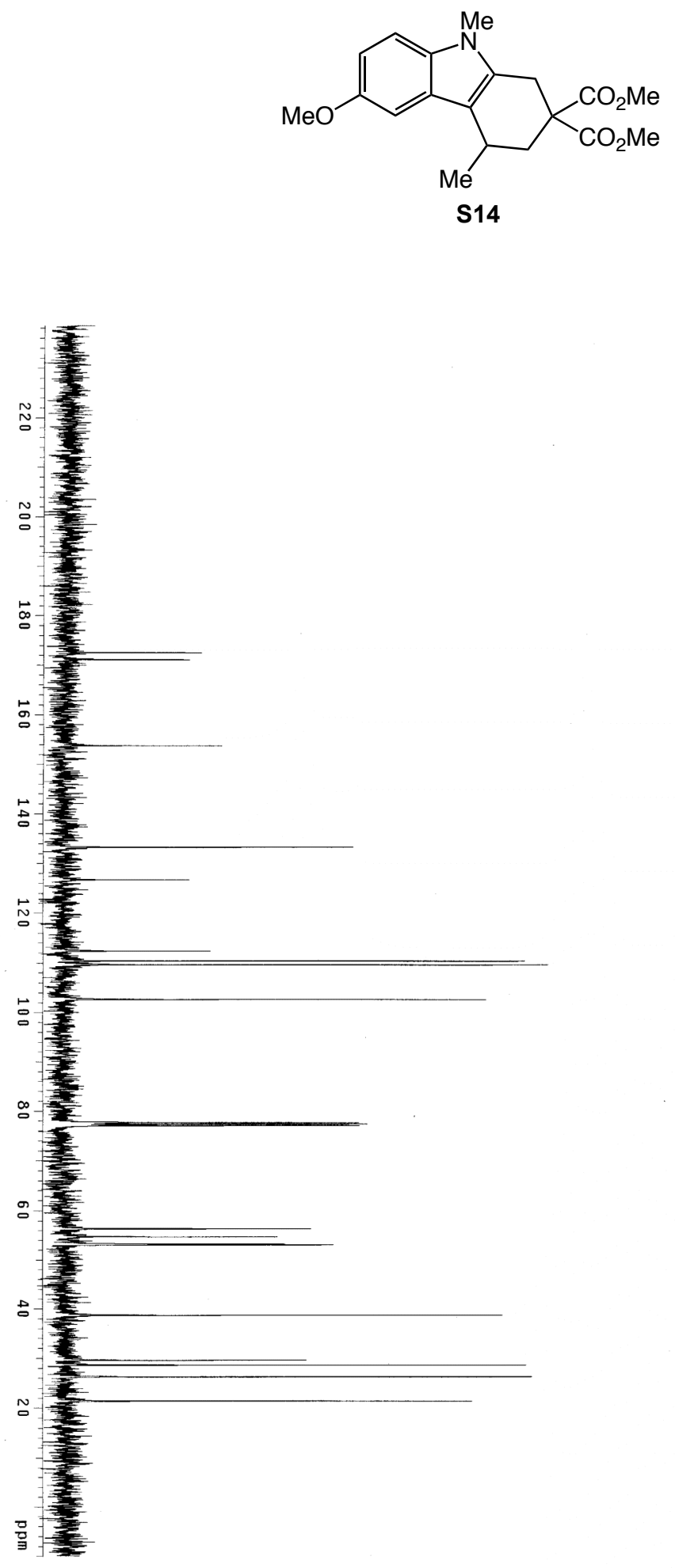
Figure S20. ${ }^{1} \mathrm{H}$ NMR spectrum of $\mathbf{S 1 5}$ in $\mathrm{CDCl}_{3}$.
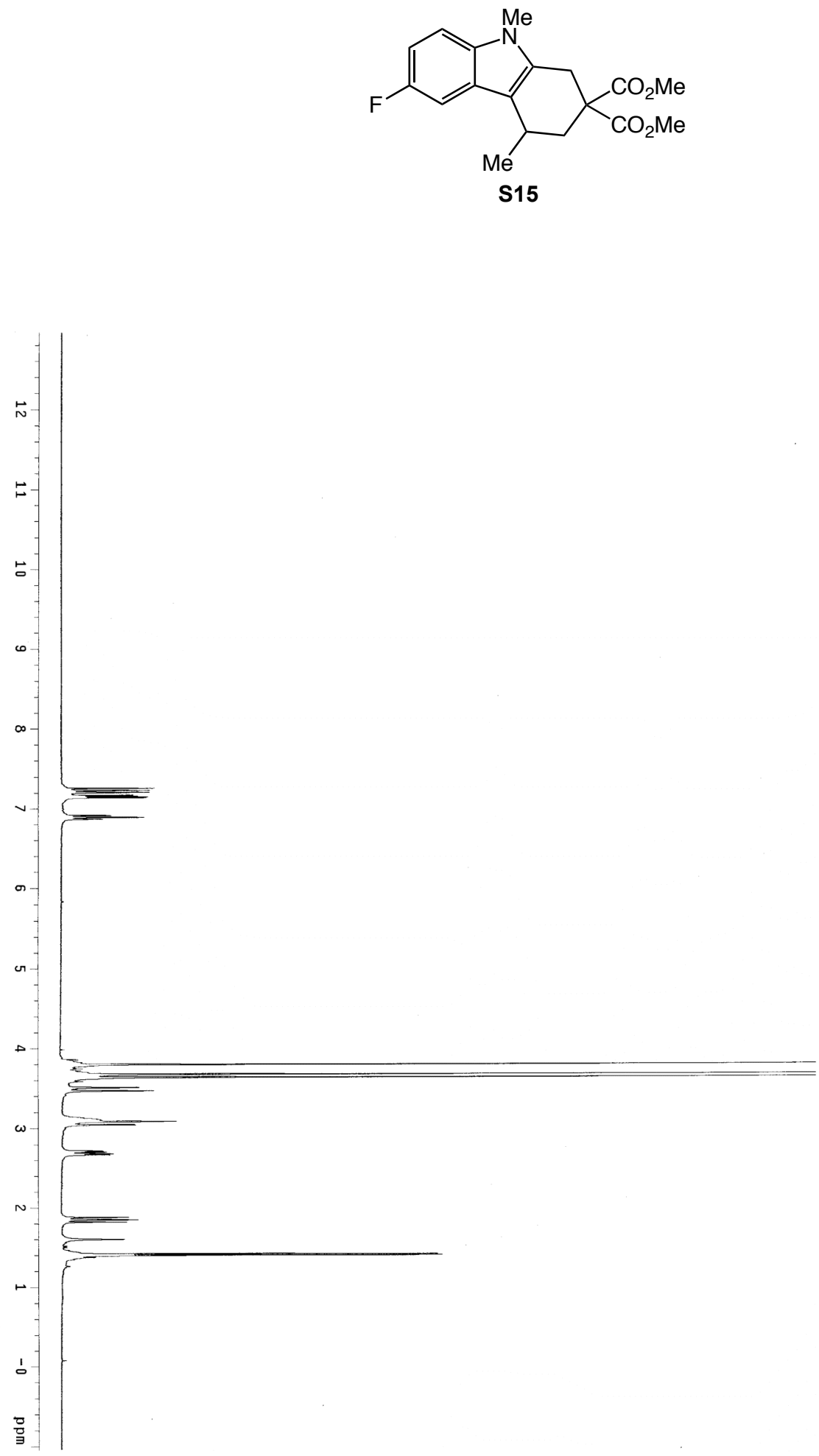
Figure S21. ${ }^{13} \mathrm{C}\left\{{ }^{1} \mathrm{H}\right\}$ NMR spectrum of $\mathbf{S 1 5}$ in $\mathrm{CDCl}_{3}$.
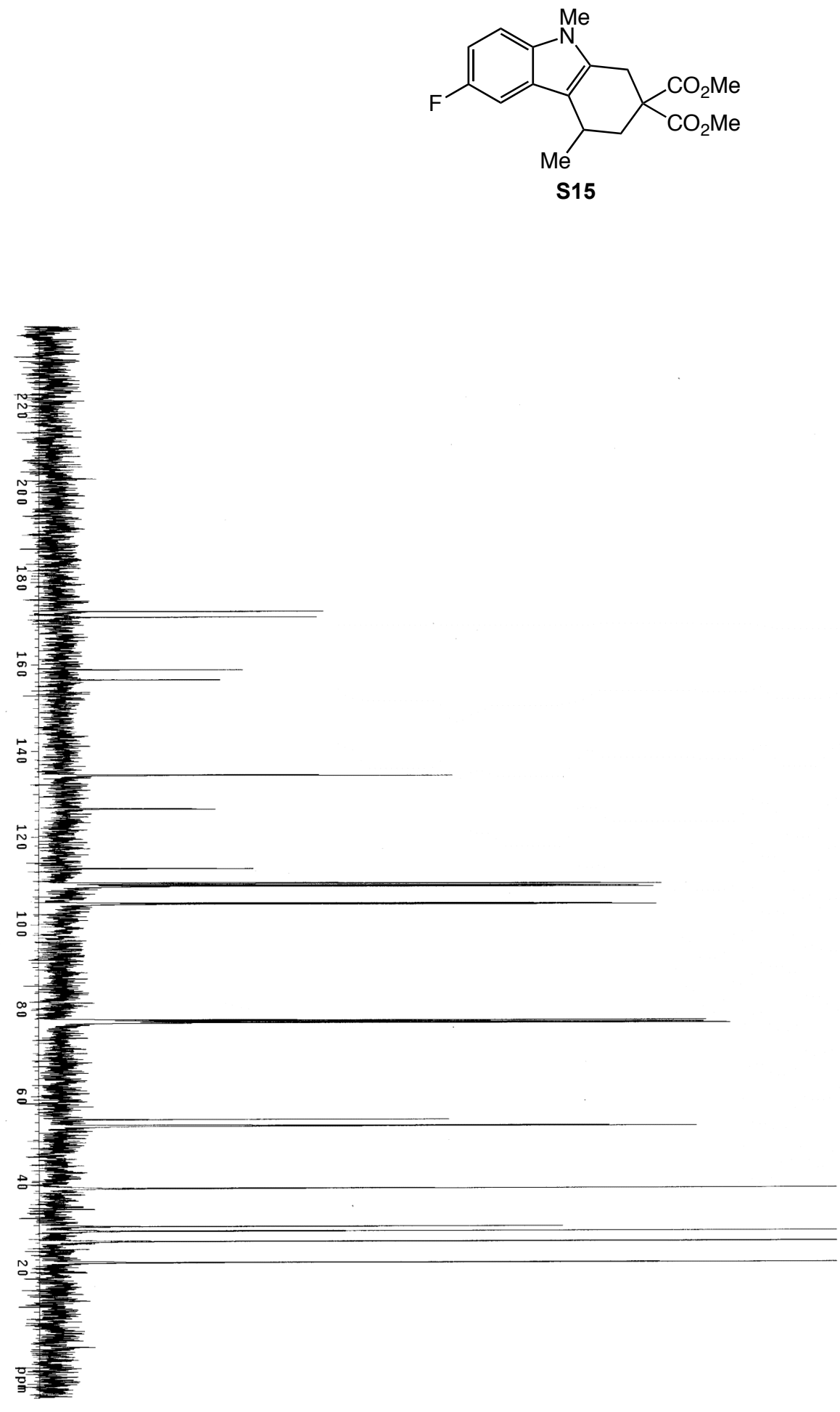
Figure S22. ${ }^{1} \mathrm{H}$ NMR spectrum of $\mathbf{S 1 6}$ in $\mathrm{CDCl}_{3}$.
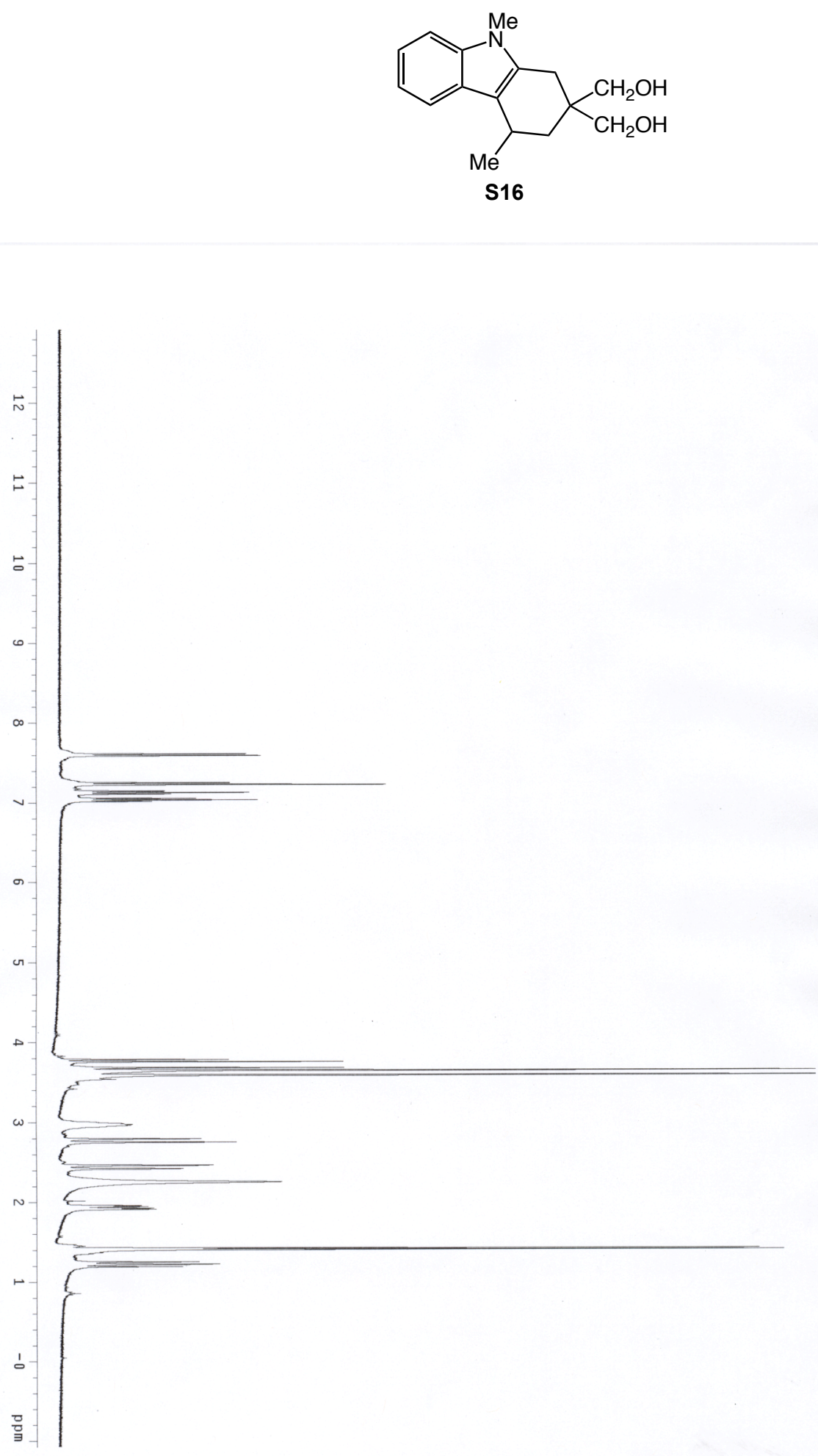
Figure S23. ${ }^{13} \mathrm{C}\left\{{ }^{1} \mathrm{H}\right\}$ NMR spectrum of $\mathbf{S 1 6}$ in $\mathrm{CDCl}_{3}$.
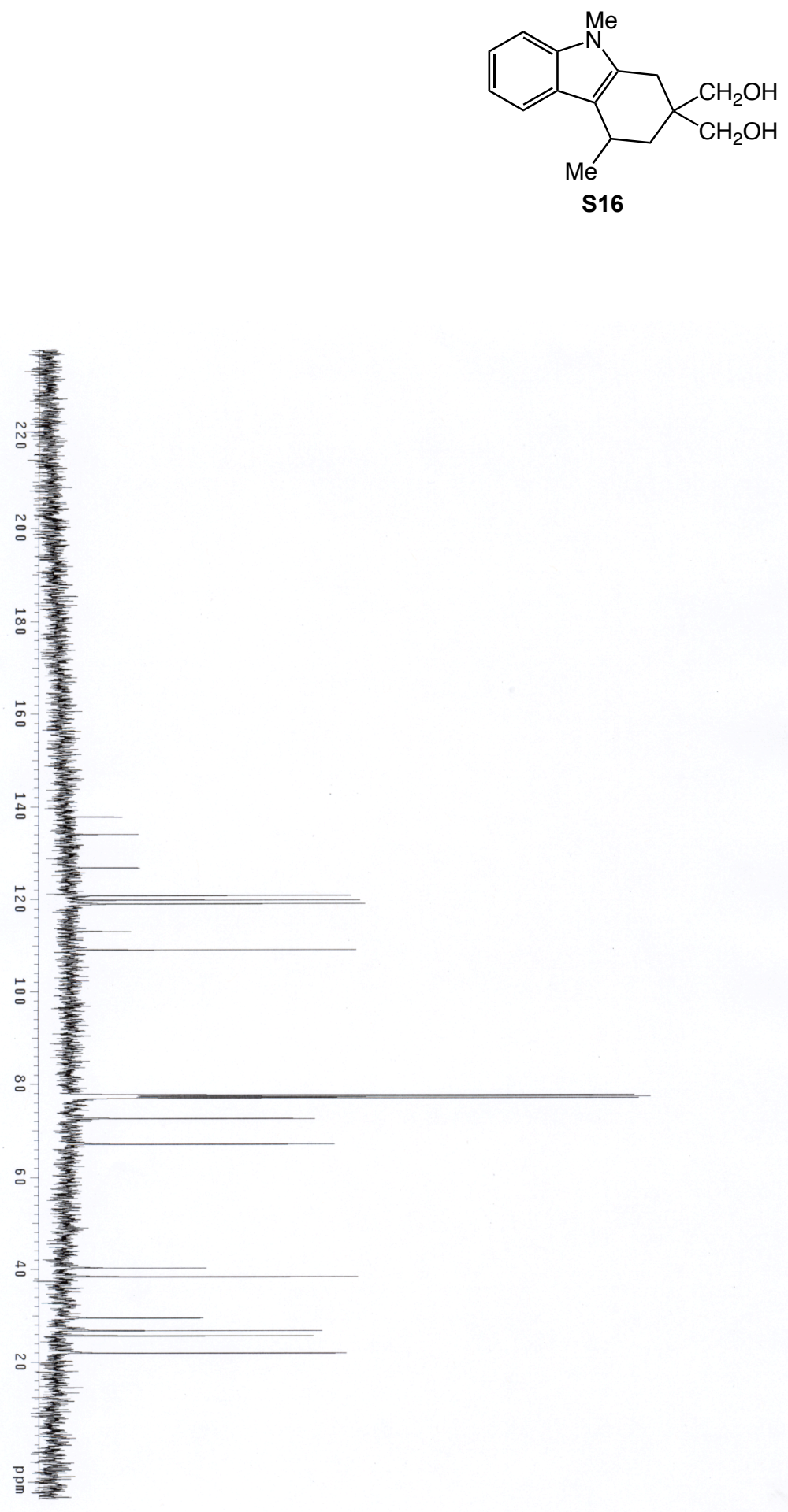
Figure S24. ${ }^{1} \mathrm{H}$ NMR spectrum of $\mathbf{S 1 7}$ in $\mathrm{CDCl}_{3}$.
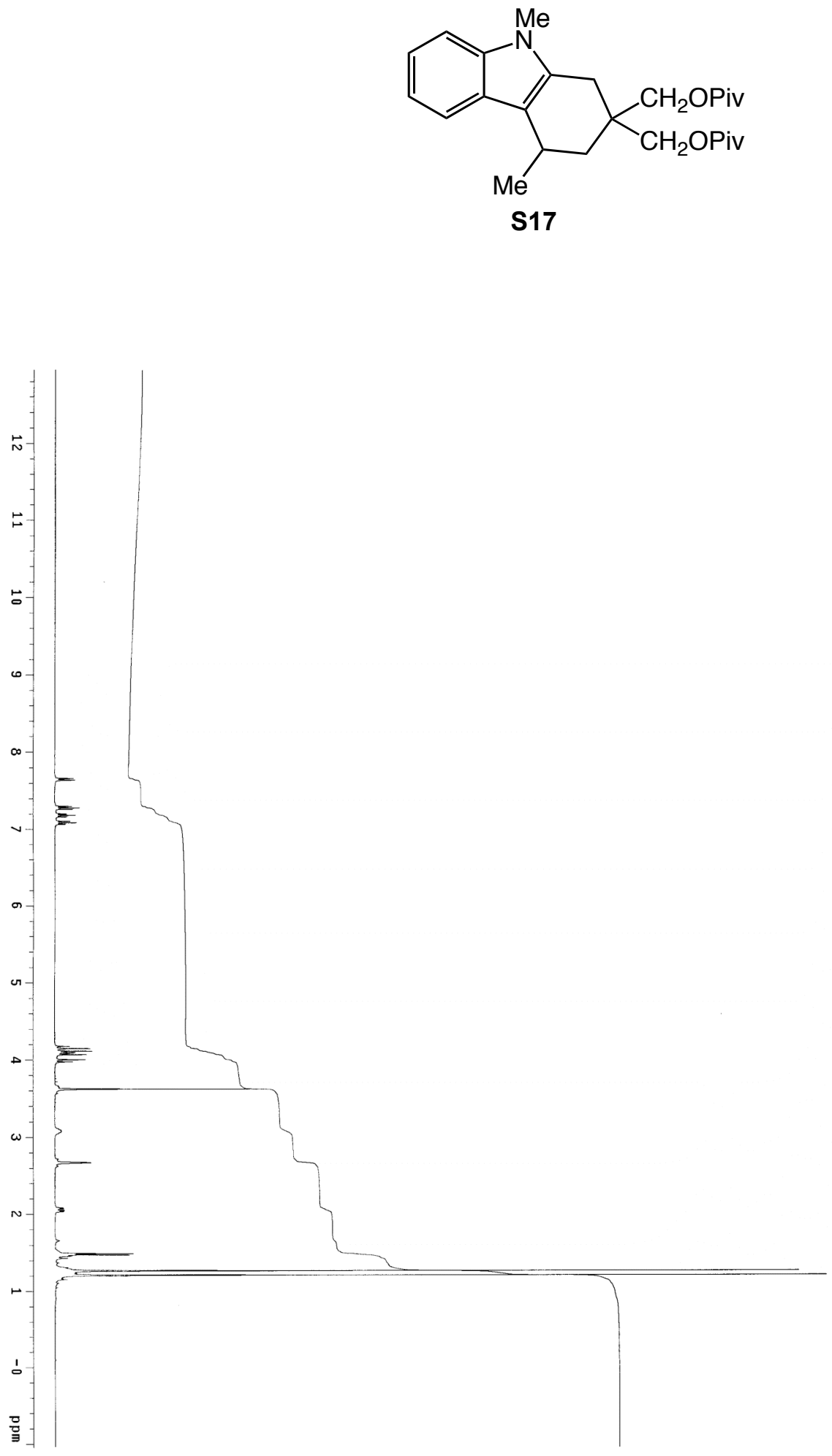
Figure S25. ${ }^{13} \mathrm{C}\left\{{ }^{1} \mathrm{H}\right\}$ NMR spectrum of $\mathbf{S 1 7}$ in $\mathrm{CDCl}_{3}$.
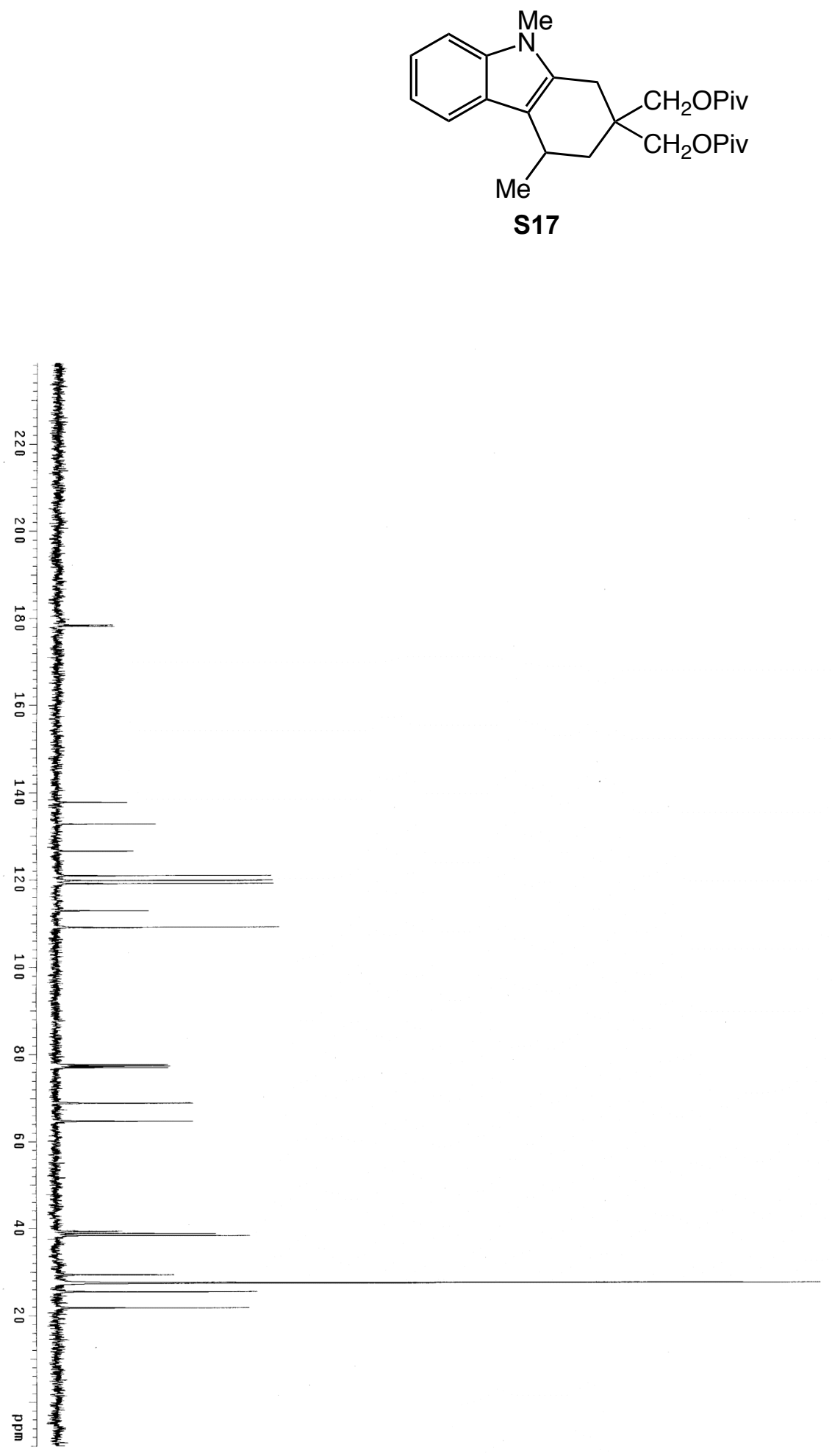
Figure S26. ${ }^{1} \mathrm{H}$ NMR spectrum of $\mathbf{S 1 8}$ in $\mathrm{CDCl}_{3}$.
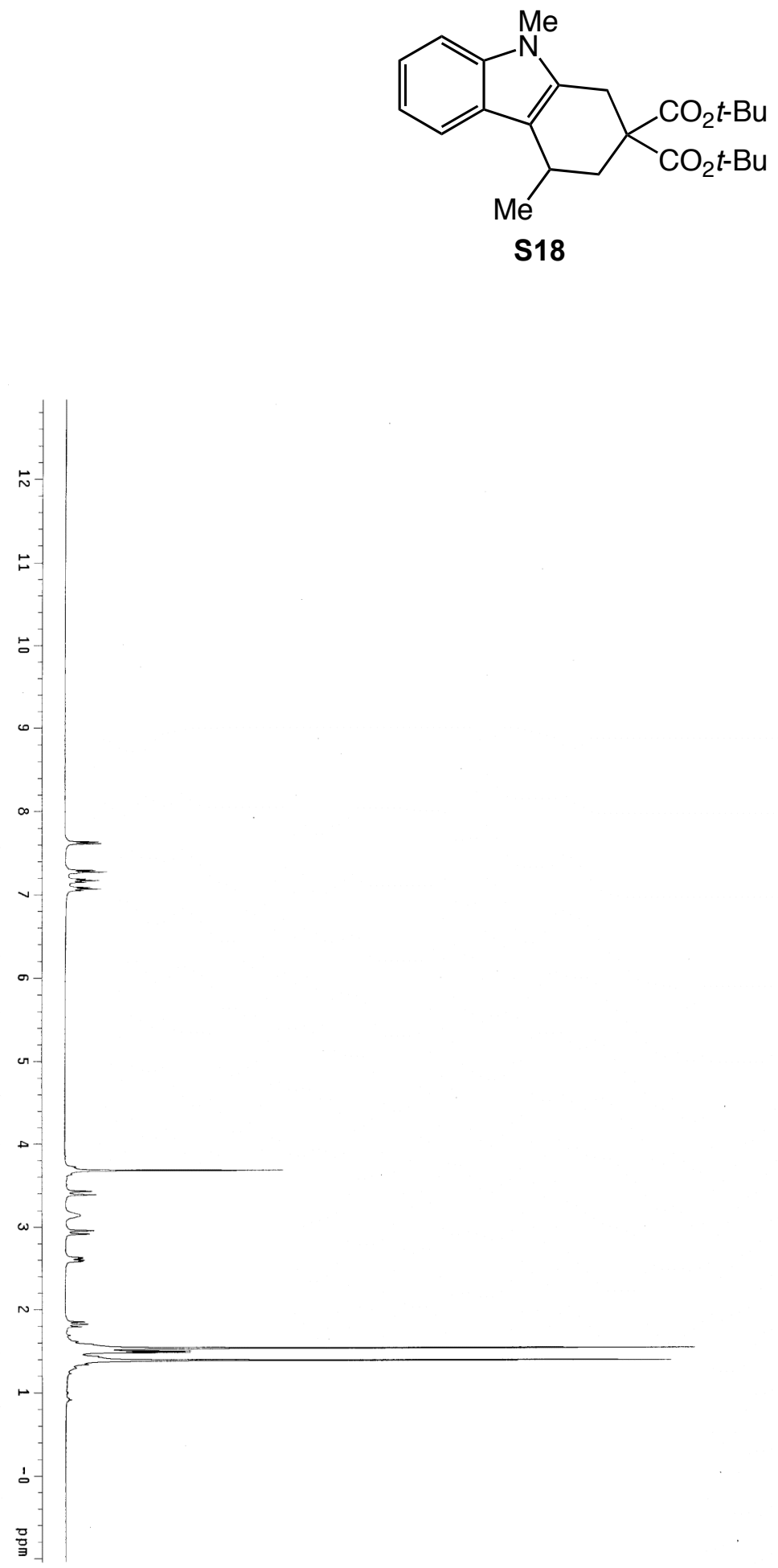


$$
\mid
$$


Figure S28. ${ }^{1} \mathrm{H}$ NMR spectrum of $\mathbf{S 1 9}$ in $\mathrm{CDCl}_{3}$.
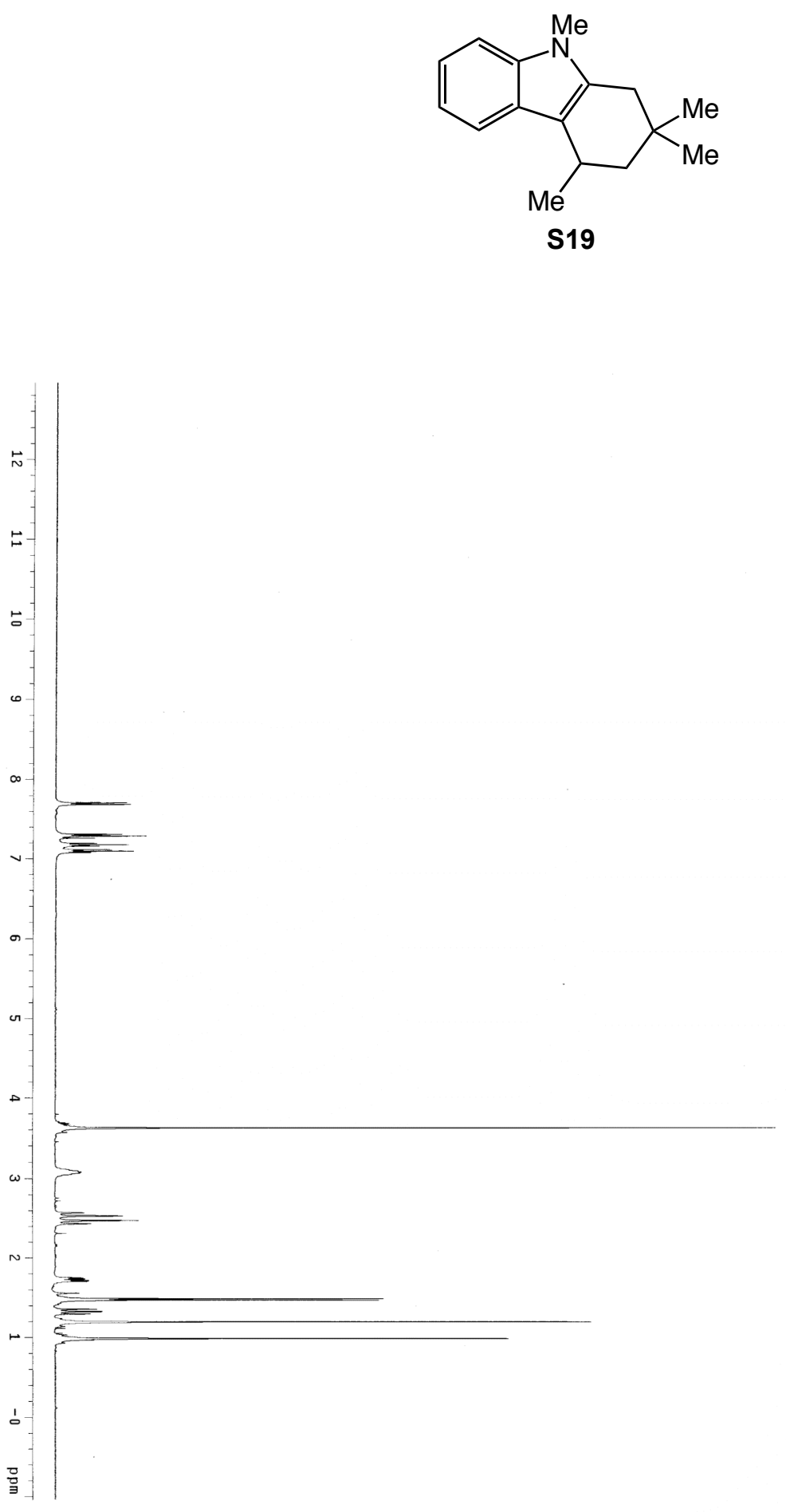


$$
E
$$


Figure S30. ${ }^{1} \mathrm{H}$ NMR spectrum of $\mathbf{S 2 0}$ in $\mathrm{CDCl}_{3}$.
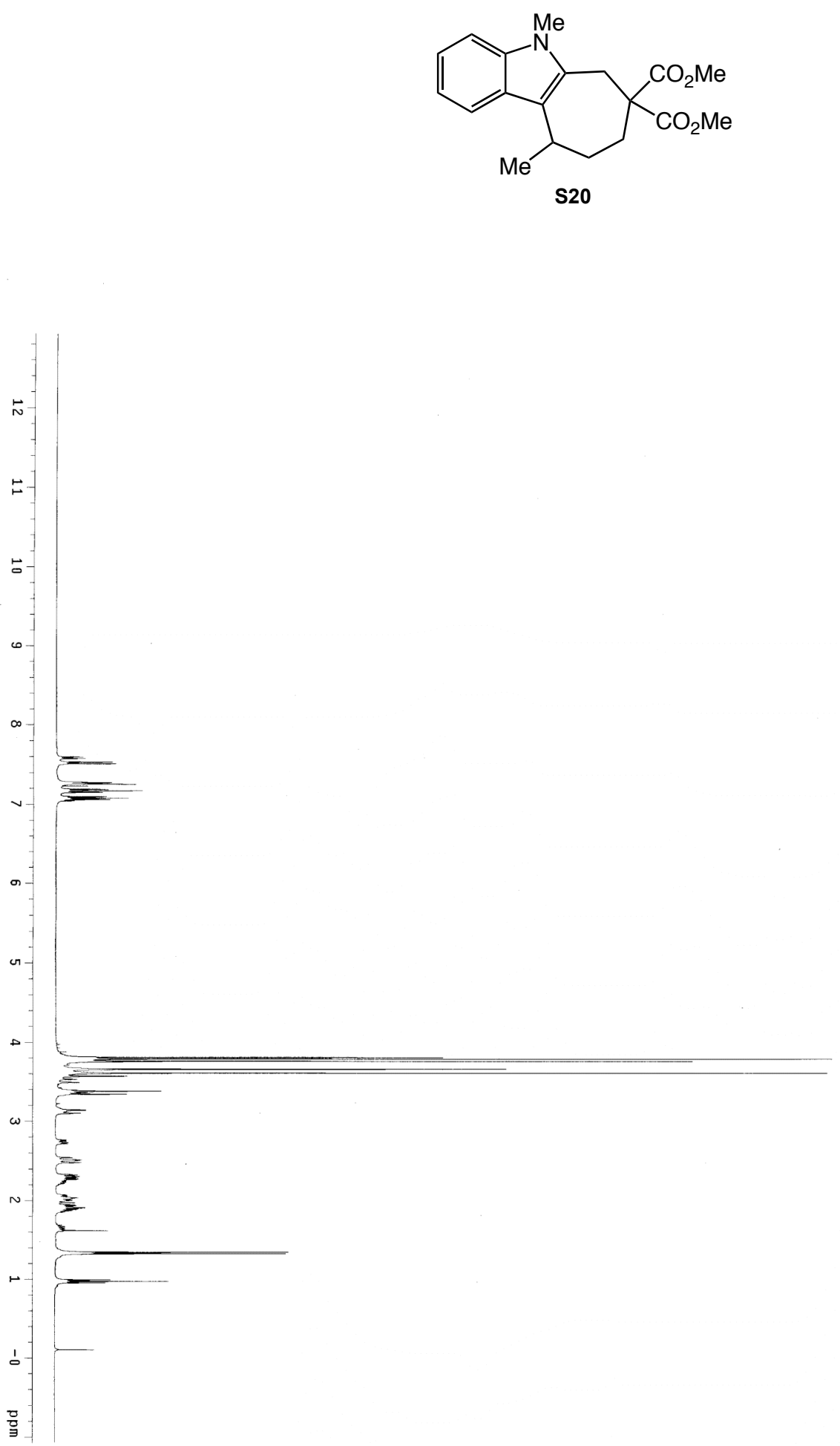


$$
1
$$


Figure S32. ${ }^{1} \mathrm{H}$ NMR spectrum for $c i s-8$ in $\mathrm{CDCl}_{3}$.

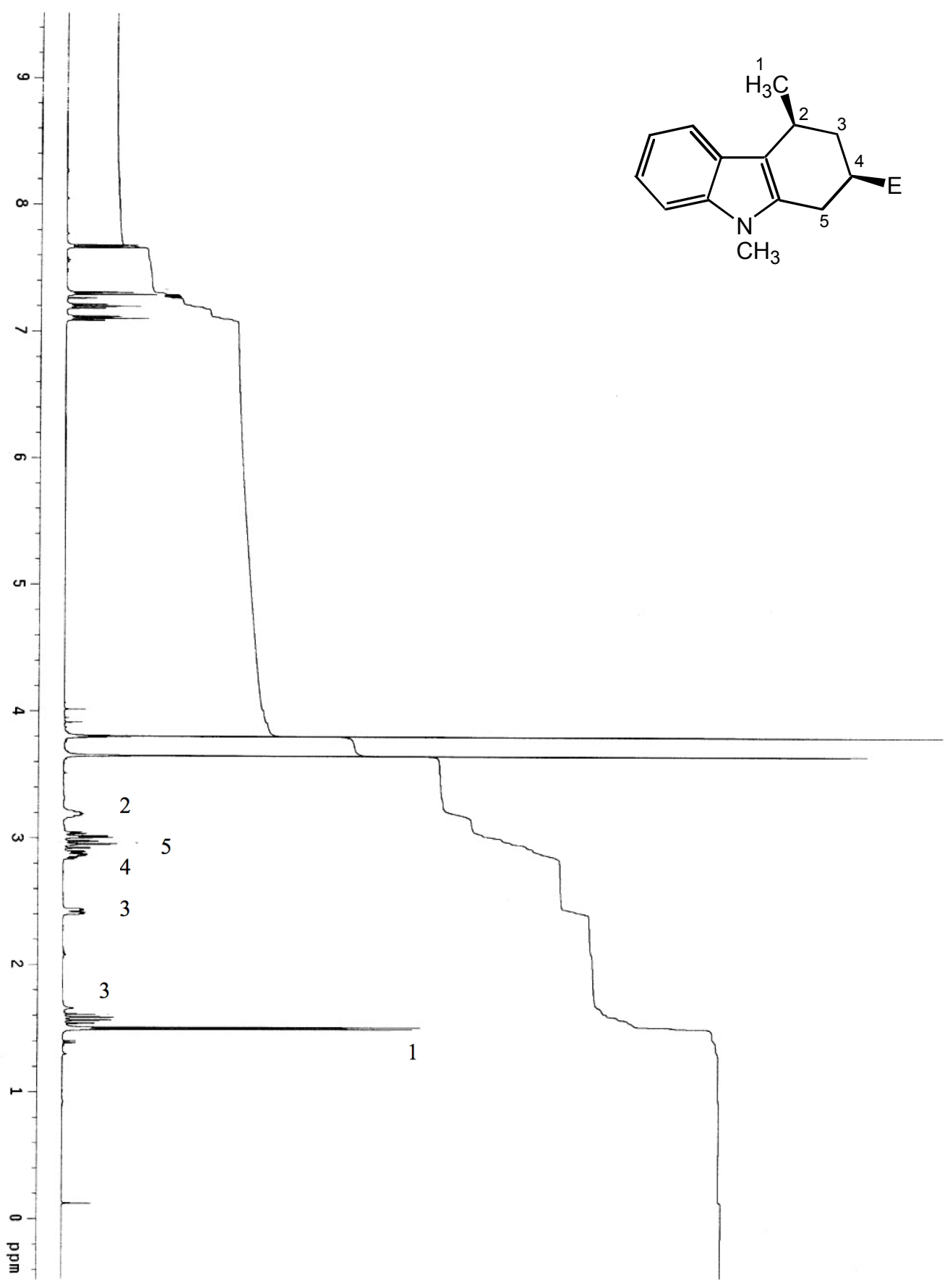


Figure S33. ${ }^{13} \mathrm{C}\left\{{ }^{1} \mathrm{H}\right\}$ NMR spectrum for $c i s-\mathbf{8}$ in $\mathrm{CDCl}_{3}$.<smiles>Cc1[nH]c2ccccc2c1C1CC(C)CC(F)C1</smiles>

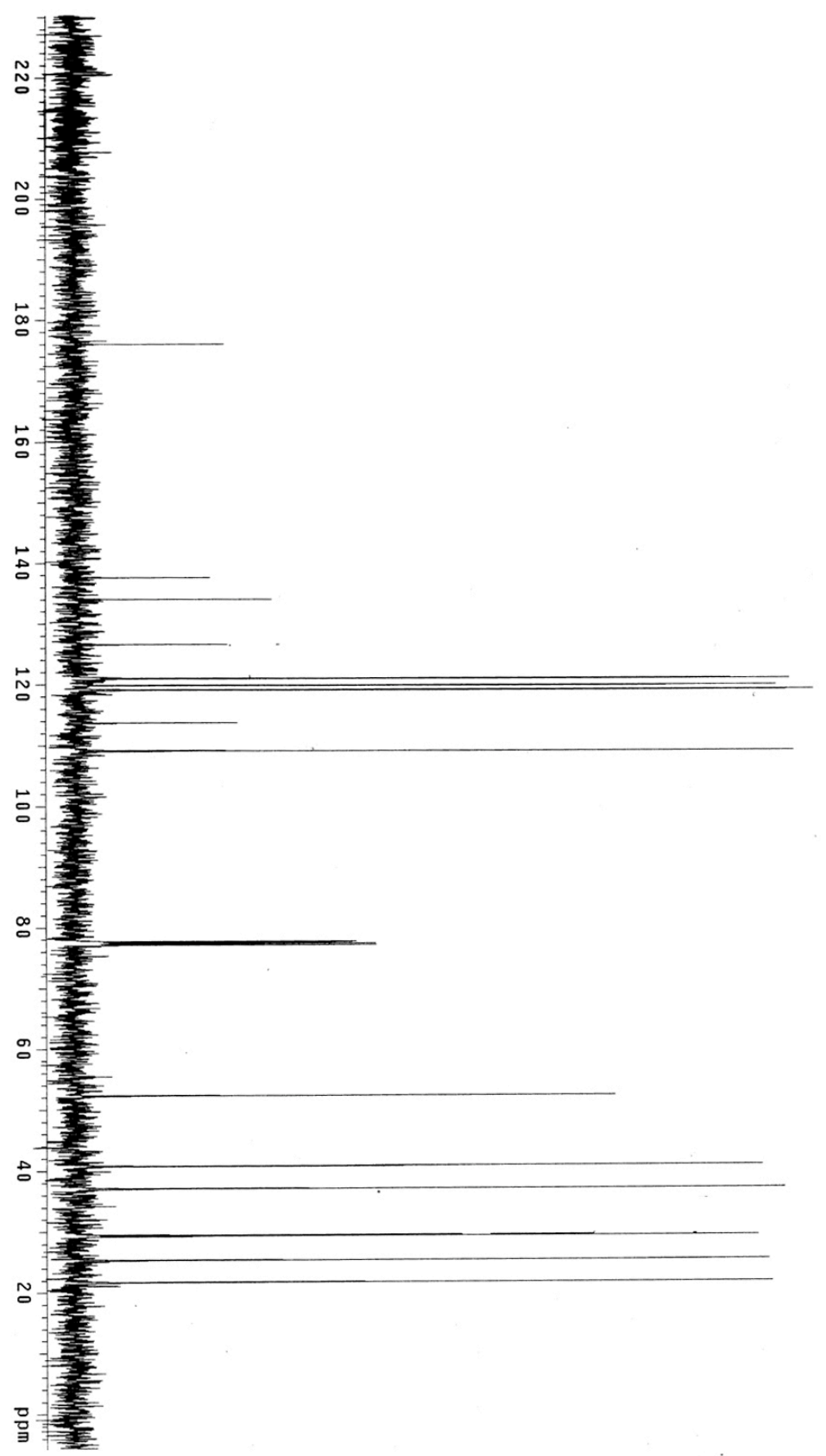


Figure S34. X-ray crystal structure of $c i s-\mathbf{S 2 2}$.

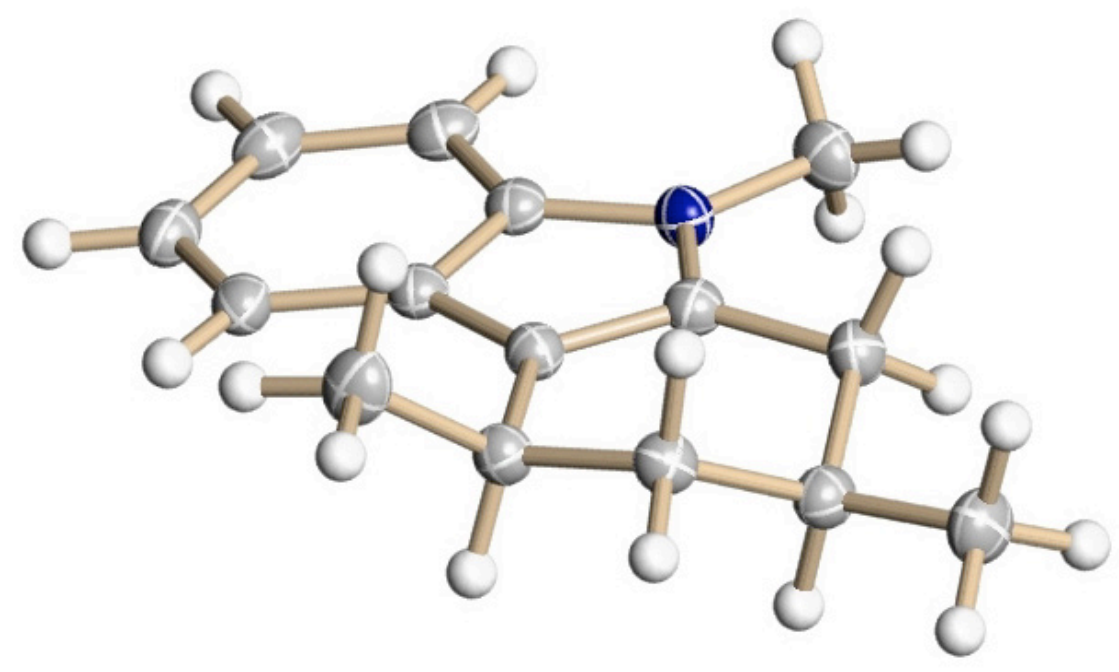

Table S1. Effect of BINAP and MeOBIPHEP ligands on platinum-catalyzed cyclization of $\mathbf{3}$ to $\mathbf{5}$ in dioxane at $60{ }^{\circ} \mathrm{C}$.

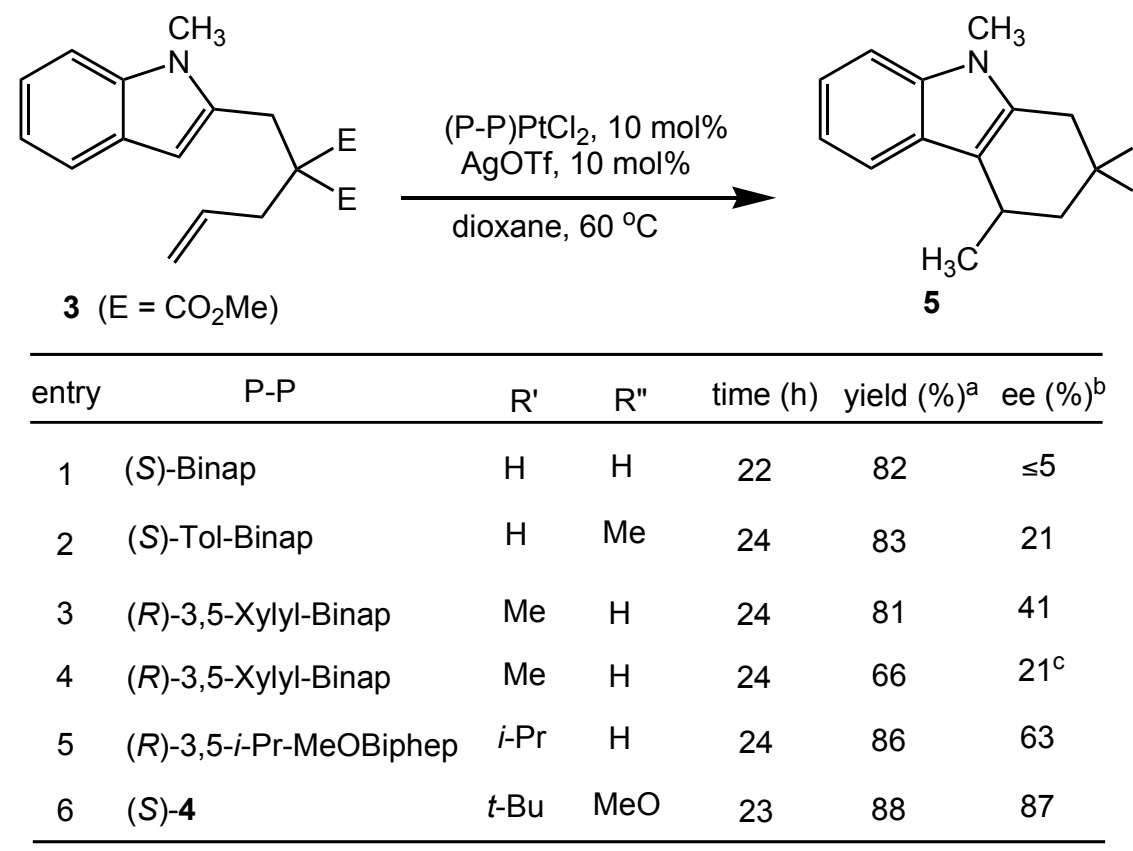

${ }^{a}$ Isolated product $\geq 95 \%$ purity. ${ }^{b}$ ee was determinated by HPLC using Chiral Pak AD-H column. ${ }^{\mathrm{c}} 20$ mol \% of AgOTf was used. 
Table S2. Effect of silver salt and solvent on cyclization of 3 to 5 catalyzed by $[(S)-4] \mathrm{PtCl}_{2}(10 \mathrm{~mol} \%)$ at $60^{\circ} \mathrm{C}$.

\begin{tabular}{|c|c|c|c|c|c|}
\hline 3 & $\left.=\mathrm{CO}_{2} \mathrm{Me}\right)$ & $\begin{array}{r}{[(S)-4] \mathrm{Pt}} \\
\operatorname{AgX}(10 \mathrm{~m} \\
\text { solvent, } 6\end{array}$ & & $-{ }^{\mathrm{CH}}$ & \\
\hline entry & solvent & $\operatorname{AgX}$ & time (h) & yield $(\%)^{a}$ & ee $(\%)^{b}$ \\
\hline 1 & 1,4-dioxane & $\mathrm{AgOTf}$ & 23 & 88 & 87 \\
\hline 2 & 1,4-dioxane & $\mathrm{AgAsF}_{6}$ & 46 & 20 & 87 \\
\hline 3 & acetone & $\mathrm{AgOTf}$ & 21 & 83 & 90 \\
\hline 4 & acetone & $\mathrm{AgSbF}_{6}$ & 18 & 84 & 88 \\
\hline 5 & $\mathrm{MeOH}$ & $\mathrm{AgOTf}$ & 20 & 93 & 90 \\
\hline 6 & $\mathrm{MeOH}$ & $\mathrm{AgBF}_{4}$ & 20 & 95 & 89 \\
\hline 7 & $t-\mathrm{BuOH}$ & AgOTf & 43 & 94 & 90 \\
\hline 8 & toluene & AgOTf & 24 & $20^{c}$ & $\sim d$ \\
\hline 9 & $\mathrm{CH}_{3} \mathrm{CN}$ & AgOTf & 24 & $7^{c}$ & $\sim d$ \\
\hline 10 & $\mathrm{Cl}_{2} \mathrm{CHCHCl}_{2}$ & AgOTf & 19 & 47 & $<5$ \\
\hline 11 & DCE & $\mathrm{AgOTf}$ & 45 & 79 & 81 \\
\hline 12 & THF & AgOTf & 23 & 92 & 89 \\
\hline 13 & $\mathrm{CH}_{2} \mathrm{Cl}_{2}$ & AgOTf & 43 & 82 & 83 \\
\hline 14 & DMF & $\mathrm{AgBF}_{4}$ & 24 & $<1^{\mathrm{c}}$ & $\sim^{d}$ \\
\hline
\end{tabular}

${ }^{\mathrm{a}}$ Isolated product $\geq 95 \%$ purity. ${ }^{\mathrm{b}}$ ee was determinated by HPLC using Chiral Pak AD-H column. ${ }^{\mathrm{c}}$ As determined by GC analysis of the crude reaction mixture. ${ }^{e}$ ee was not determined. 
Table S3. X-ray crystal data and refinement for $c i s-\mathbf{S 2 2}$.

\begin{tabular}{ll}
\hline empirical formula & $\mathrm{C}_{15} \mathrm{H}_{19} \mathrm{~N}$ \\
fw & 213.31 \\
Temp (K) & $98(2)$ \\
Wavelength & $0.71073 \AA$ \\
crystal size, mm & $0.40 \times 0.17 \times 0.14$ \\
crystal system & monoclinic \\
color and habit & colorless needle \\
space group & $P 21 / \mathrm{n}$ \\
$a, \AA$ & $13.6855(5)$ \\
$b, \AA$ & $28.583(1)$ \\
$c, \AA$ & $14.6784(5)$ \\
$\mathrm{V}, \AA^{3}$ & $1174.9(5)$ \\
$Z$ & 4 \\
scan mode & $\omega$ \\
$2 \theta$ limits $($ deg $)$ & $3.62 \leq 2 \theta \leq 31.81$ \\
$D_{\mathrm{c}}(\mathrm{g}$ cm & -3 \\
abs. coeff. $\left(\mathrm{mm}{ }^{-1}\right)$ & 1.206 \\
reflections & 0.069 \\
unique reflections & 12477 \\
data with $/>2.5 \sigma(I)$ & 3730 \\
$F(000)$ & 2818 \\
$R(\mathrm{~F})$ & 464 \\
$R_{\mathrm{w}}(\mathrm{F})$ & 0.0623 \\
GoF & 0.1279 \\
no. param & 0.979 \\
max $\Delta / \sigma$ & 221 \\
largest res. density $\left(\mathrm{e} / \AA^{3}\right)$ & 0.18 \\
\hline
\end{tabular}


Table S4. Atomic coordinates $\left(\times 10^{4}\right)$ and equivalent isotropic displacement parameters $\left(\AA^{2} \times 10^{3}\right)$ for cis-S22. $\mathrm{U}_{(\mathrm{eq})}$ is defined as one third of the trace of the orthogonalized $\mathrm{U}^{\mathrm{ij}}$ tensor.

$$
\begin{array}{llll}
\mathrm{x} & \mathrm{z} & \mathrm{U} & \mathrm{eq})
\end{array}
$$

\begin{tabular}{lllll}
\hline $\mathrm{N}(1)$ & $2494(1)$ & $5261(1)$ & $-60(1)$ & $20(1)$ \\
$\mathrm{C}(12)$ & $881(1)$ & $7809(1)$ & $796(1)$ & $20(1)$ \\
$\mathrm{C}(6)$ & $2264(1)$ & $5682(1)$ & $-1113(1)$ & $19(1)$ \\
$\mathrm{C}(5)$ & $1634(1)$ & $6701(1)$ & $-996(1)$ & $18(1)$ \\
$\mathrm{C}(7)$ & $2018(1)$ & $5987(1)$ & $715(1)$ & $19(1)$ \\
$\mathrm{C}(13)$ & $3013(2)$ & $4208(1)$ & $178(1)$ & $25(1)$ \\
$\mathrm{C}(9)$ & $1993(1)$ & $5788(1)$ & $1942(1)$ & $21(1)$ \\
$\mathrm{C}(11)$ & $1282(1)$ & $7678(1)$ & $2056(1)$ & $21(1)$ \\
$\mathrm{C}(1)$ & $2547(1)$ & $5249(1)$ & $-2158(1)$ & $23(1)$ \\
$\mathrm{C}(10)$ & $861(1)$ & $6610(1)$ & $2487(1)$ & $22(1)$ \\
$\mathrm{C}(8)$ & $1520(1)$ & $6881(1)$ & $183(1)$ & $18(1)$ \\
$\mathrm{C}(3)$ & $1448(1)$ & $6829(1)$ & $-3006(1)$ & $25(1)$ \\
$\mathrm{C}(4)$ & $1215(1)$ & $7268(1)$ & $-1971(1)$ & $22(1)$ \\
$\mathrm{C}(15)$ & $1663(2)$ & $8819(1)$ & $401(1)$ & $28(1)$ \\
$\mathrm{C}(14)$ & $1065(2)$ & $6548(1)$ & $3759(1)$ & $28(1)$ \\
$\mathrm{C}(2)$ & $2121(1)$ & $5834(1)$ & $-3099(1)$ & $25(1)$ \\
& & & & \\
\hline
\end{tabular}


Table S5. Bond lengths $[\AA]$ and angles $\left[{ }^{\circ}\right]$ for $c i s-\mathbf{S 2 2}$.

\begin{tabular}{lllll}
\hline $\mathrm{N}(1)-\mathrm{C}(6)$ & $1.3743(12)$ & & \\
$\mathrm{N}(1)-\mathrm{C}(7)$ & $1.3829(12)$ & & \\
$\mathrm{N}(1)-\mathrm{C}(13)$ & $1.4489(13)$ & & \\
$\mathrm{C}(12)-\mathrm{C}(8)$ & $1.5041(13)$ & & \\
$\mathrm{C}(12)-\mathrm{C}(15)$ & $1.5252(15)$ & & \\
$\mathrm{C}(12)-\mathrm{C}(11)$ & $1.5288(15)$ & & \\
$\mathrm{C}(6)-\mathrm{C}(1)$ & $1.3956(14)$ & & \\
$\mathrm{C}(6)-\mathrm{C}(5)$ & $1.4176(14)$ & & \\
$\mathrm{C}(5)-\mathrm{C}(4)$ & $1.4008(14)$ & & \\
$\mathrm{C}(5)-\mathrm{C}(8)$ & $1.4341(14)$ & & \\
$\mathrm{C}(7)-\mathrm{C}(8)$ & $1.3687(14)$ & & \\
$\mathrm{C}(7)-\mathrm{C}(9)$ & $1.4887(14)$ & & \\
$\mathrm{C}(9)-\mathrm{C}(10)$ & $1.5361(15)$ & & \\
$\mathrm{C}(11)-\mathrm{C}(10)$ & $1.5210(15)$ & & \\
$\mathrm{C}(1)-\mathrm{C}(2)$ & $1.3821(15)$ & & \\
$\mathrm{C}(10)-\mathrm{C}(14)$ & $1.5215(15)$ & & \\
$\mathrm{C}(3)-\mathrm{C}(4)$ & $1.3811(15)$ & & \\
$\mathrm{C}(3)-\mathrm{C}(2)$ & $1.3966(16)$ & & \\
& & & & \\
$\mathrm{C}(6)-\mathrm{N}(1)-\mathrm{C}(7)$ & $108.40(8)$ & $\mathrm{C}(6)-\mathrm{N}(1)-\mathrm{C}(13)$ & $125.20(9)$ \\
$\mathrm{C}(7)-\mathrm{N}(1)-\mathrm{C}(13)$ & $126.23(9)$ & $\mathrm{C}(8)-\mathrm{C}(12)-\mathrm{C}(15)$ & $113.60(9)$ \\
$\mathrm{C}(8)-\mathrm{C}(12)-\mathrm{C}(11)$ & $109.70(8)$ & $\mathrm{C}(15)-\mathrm{C}(12)-\mathrm{C}(11)$ & $109.87(8)$ \\
$\mathrm{N}(1)-\mathrm{C}(6)-\mathrm{C}(1)$ & $129.72(10)$ & $\mathrm{N}(1)-\mathrm{C}(6)-\mathrm{C}(5)$ & $108.01(8)$ \\
$\mathrm{C}(1)-\mathrm{C}(6)-\mathrm{C}(5)$ & $122.27(9)$ & $\mathrm{C}(4)-\mathrm{C}(5)-\mathrm{C}(6)$ & $118.18(9)$ \\
$\mathrm{C}(4)-\mathrm{C}(5)-\mathrm{C}(8)$ & $135.06(9)$ & $\mathrm{C}(6)-\mathrm{C}(5)-\mathrm{C}(8)$ & $106.71(8)$ \\
$\mathrm{C}(8)-\mathrm{C}(7)-\mathrm{N}(1)$ & $110.13(9)$ & $\mathrm{C}(8)-\mathrm{C}(7)-\mathrm{C}(9)$ & $125.93(9)$ \\
$\mathrm{N}(1)-\mathrm{C}(7)-\mathrm{C}(9)$ & $123.89(9)$ & $\mathrm{C}(7)-\mathrm{C}(9)-\mathrm{C}(10)$ & $109.42(8)$ \\
$\mathrm{C}(10)-\mathrm{C}(11)-\mathrm{C}(12)$ & $113.60(8)$ & $\mathrm{C}(2)-\mathrm{C}(1)-\mathrm{C}(6)$ & $117.67(10)$ \\
$\mathrm{C}(11)-\mathrm{C}(10)-\mathrm{C}(14)$ & $111.97(9)$ & $\mathrm{C}(11)-\mathrm{C}(10)-\mathrm{C}(9)$ & $111.12(8)$ \\
$\mathrm{C}(14)-\mathrm{C}(10)-\mathrm{C}(9)$ & $110.90(9)$ & $\mathrm{C}(7)-\mathrm{C}(8)-\mathrm{C}(5)$ & $106.72(8)$ \\
$\mathrm{C}(7)-\mathrm{C}(8)-\mathrm{C}(12)$ & $122.93(9)$ & $\mathrm{C}(5)-\mathrm{C}(8)-\mathrm{C}(12)$ & $130.23(9)$ \\
$\mathrm{C}(4)-\mathrm{C}(3)-\mathrm{C}(2)$ & $121.17(10)$ & $\mathrm{C}(3)-\mathrm{C}(4)-\mathrm{C}(5)$ & $119.55(10)$ \\
$\mathrm{C}(1)-\mathrm{C}(2)-\mathrm{C}(3)$ & $121.09(10)$ & & \\
& & & & \\
\hline & & & \\
\end{tabular}


Table S6. Anisotropic displacement parameters $\left(\AA^{2} \times 10^{3}\right)$ for cis-S22. The anisotropic displacement factor exponent takes the form: $-2 \pi^{2}\left[h^{2} \mathrm{a}^{*^{2}} \mathrm{U}^{11}+2 h k \mathrm{a}^{*} \mathrm{~b}^{*} \mathrm{U}^{12}\right]$.

\begin{tabular}{lllllll}
\hline & $\mathrm{U}^{11}$ & $\mathrm{U}^{22}$ & $\mathrm{U}^{33}$ & $\mathrm{U}^{23}$ & $\mathrm{U}^{13}$ & $\mathrm{U}^{12}$ \\
\hline $\mathrm{N}(1)$ & $22(1)$ & $17(1)$ & $20(1)$ & $0(1)$ & $1(1)$ & $1(1)$ \\
$\mathrm{C}(12)$ & $21(1)$ & $19(1)$ & $20(1)$ & $-1(1)$ & $1(1)$ & $1(1)$ \\
$\mathrm{C}(6)$ & $16(1)$ & $19(1)$ & $21(1)$ & $0(1)$ & $1(1)$ & $-3(1)$ \\
$\mathrm{C}(5)$ & $15(1)$ & $19(1)$ & $20(1)$ & $0(1)$ & $1(1)$ & $-2(1)$ \\
$\mathrm{C}(7)$ & $17(1)$ & $19(1)$ & $19(1)$ & $-1(1)$ & $0(1)$ & $-1(1)$ \\
$\mathrm{C}(13)$ & $27(1)$ & $18(1)$ & $29(1)$ & $1(1)$ & $-1(1)$ & $2(1)$ \\
$\mathrm{C}(9)$ & $23(1)$ & $21(1)$ & $20(1)$ & $2(1)$ & $-1(1)$ & $0(1)$ \\
$\mathrm{C}(11)$ & $22(1)$ & $22(1)$ & $20(1)$ & $-3(1)$ & $0(1)$ & $0(1)$ \\
$\mathrm{C}(1)$ & $20(1)$ & $22(1)$ & $26(1)$ & $-4(1)$ & $4(1)$ & $-4(1)$ \\
$\mathrm{C}(10)$ & $22(1)$ & $25(1)$ & $19(1)$ & $0(1)$ & $1(1)$ & $-1(1)$ \\
$\mathrm{C}(8)$ & $17(1)$ & $18(1)$ & $19(1)$ & $0(1)$ & $1(1)$ & $-1(1)$ \\
$\mathrm{C}(3)$ & $24(1)$ & $30(1)$ & $20(1)$ & $3(1)$ & $1(1)$ & $-5(1)$ \\
$\mathrm{C}(4)$ & $20(1)$ & $23(1)$ & $22(1)$ & $3(1)$ & $1(1)$ & $-2(1)$ \\
$\mathrm{C}(15)$ & $37(1)$ & $18(1)$ & $30(1)$ & $-1(1)$ & $5(1)$ & $0(1)$ \\
$\mathrm{C}(14)$ & $34(1)$ & $33(1)$ & $19(1)$ & $1(1)$ & $1(1)$ & $-1(1)$ \\
$\mathrm{C}(2)$ & $25(1)$ & $30(1)$ & $21(1)$ & $-5(1)$ & $4(1)$ & $-7(1)$ \\
& & & & & & \\
\hline
\end{tabular}


Table S7. Hydrogen coordinates $\left(\times 10^{4}\right)$ and isotropic displacement parameters for cis-S22.

\begin{tabular}{lllll}
\hline & \multicolumn{1}{c}{$\mathrm{x}$} & \multicolumn{1}{c}{$\mathrm{y}$} & $\mathrm{U}(\mathrm{eq})$ \\
& & & & \\
$\mathrm{H}(1)$ & $3005(17)$ & $4565(11)$ & $-2215(10)$ & $28(3)$ \\
$\mathrm{H}(4)$ & $765(16)$ & $7988(10)$ & $-1929(10)$ & $26(3)$ \\
$\mathrm{H}(9 \mathrm{~B})$ & $1523(18)$ & $5093(11)$ & $2117(11)$ & $35(4)$ \\
$\mathrm{H}(2)$ & $2292(17)$ & $5545(11)$ & $-3829(11)$ & $35(4)$ \\
$\mathrm{H}(11 \mathrm{~A})$ & $2547(17)$ & $7818(9)$ & $2222(10)$ & $23(3)$ \\
$\mathrm{H}(3)$ & $1147(16)$ & $7227(10)$ & $-3676(10)$ & $26(3)$ \\
$\mathrm{H}(9 \mathrm{~A})$ & $3226(17)$ & $5805(10)$ & $2304(10)$ & $26(3)$ \\
$\mathrm{H}(12)$ & $-437(16)$ & $7844(9)$ & $646(10)$ & $21(3)$ \\
$\mathrm{H}(15 \mathrm{~B})$ & $2980(20)$ & $8826(11)$ & $542(13)$ & $44(4)$ \\
$\mathrm{H}(15 \mathrm{C})$ & $1224(18)$ & $9405(11)$ & $823(11)$ & $32(3)$ \\
$\mathrm{H}(14 \mathrm{~A})$ & $822(18)$ & $5841(11)$ & $4041(11)$ & $31(3)$ \\
$\mathrm{H}(11 \mathrm{~B})$ & $562(17)$ & $8192(10)$ & $2469(10)$ & $24(3)$ \\
$\mathrm{H}(15 \mathrm{~A})$ & $1360(20)$ & $8978(11)$ & $-410(13)$ & $44(4)$ \\
$\mathrm{H}(10)$ & $-431(16)$ & $6472(9)$ & $2226(10)$ & $22(3)$ \\
$\mathrm{H}(14 \mathrm{~B})$ & $2290(20)$ & $6709(11)$ & $4050(11)$ & $37(4)$ \\
$\mathrm{H}(13 \mathrm{~B})$ & $2150(20)$ & $3732(13)$ & $-81(14)$ & $60(5)$ \\
$\mathrm{H}(14 \mathrm{C})$ & $207(19)$ & $7056(12)$ & $4108(12)$ & $40(4)$ \\
$\mathrm{H}(13 \mathrm{C})$ & $3410(30)$ & $4133(15)$ & $939(17)$ & $76(6)$ \\
$\mathrm{H}(13 \mathrm{~A})$ & $3930(30)$ & $3979(13)$ & $-340(15)$ & $68(5)$ \\
& & & & \\
\hline
\end{tabular}




\section{References}

S1) Liu, C.; Han, X.; Wang, X.; Widenhoefer, R. A., J. Am. Chem. Soc. 2004, 126, 3700.

S2) Strukul, G.; Varagnolo, A.; Pinna, F., J Mol. Catal. A-Chem. 1997, 117, 413.

S3) Beckwith, A. L.J.; Bowry, V. W.; Moad, G. J. Org. Chem. 1988, 53, 1632.

S4) Hlasta, K. J.; Luttinger, D.; Perrone, M.. H.; Silibernagle, M. J.; Ward, S. J.; Saubricht, D. R. J. Med. Chem. 1987, 30, 1555.

S5) Fernandez-Garcia, C.; Marco, J. L.; Fernandez-Alverez, E. Eur. J. Med. Chem. 1992, $27,909$.

S6) Smith, A. B. L.; Visnick, M.; Hasletine, J. N.; Sprengeler, P. A. Tetrahedron 1986, 42, 2957. 